\title{
Correlation between small-scale velocity and scalar fluctuations in a turbulent channel flow
}

\author{
HIROYUKI ABE ${ }^{1} \dagger$, ROBERT ANTHONY ANTONIA ${ }^{2}$ \\ AND HIROSHI KA WAMURA ${ }^{3}$ \\ ${ }^{1}$ Japan Aerospace Exploration Agency, 182-8522 Tokyo, Japan \\ ${ }^{2}$ Discipline of Mechanical Engineering, University of Newcastle, 2308 NSW, Australia \\ ${ }^{3}$ Department of Mechanical Engineering, Tokyo University of Science, 278-8510 Chiba, Japan
}

(Received 7 May 2008 and in revised form 11 December 2008)

Direct numerical simulations of a turbulent channel flow with passive scalar transport are used to examine the relationship between small-scale velocity and scalar fields. The Reynolds number based on the friction velocity and the channel half-width is equal to 180,395 and 640 , and the molecular Prandtl number is 0.71 . The focus is on the interrelationship between the components of the vorticity vector and those of the scalar derivative vector. Near the wall, there is close similarity between different components of the two vectors due to the almost perfect correspondence between the momentum and thermal streaks. With increasing distance from the wall, the magnitudes of the correlations become smaller but remain non-negligible everywhere in the channel owing to the presence of internal shear and scalar layers in the inner region and the backs of the large-scale motions in the outer region. The topology of the scalar dissipation rate, which is important for small-scale scalar mixing, is shown to be associated with the organized structures. The most preferential orientation of the scalar dissipation rate is the direction of the mean strain rate near the wall and that of the fluctuating compressive strain rate in the outer region. The latter region has many characteristics in common with several turbulent flows; viz. the dominant structures are sheetlike in form and better correlated with the energy dissipation rate than the enstrophy.

\section{Introduction}

The ability to mix scalar contaminants is one of the major characteristics of turbulence (Antonia \& Orlandi 2003). The large scales play a major part in transporting the scalar, whereas the small scales are instrumental in implementing the mixing at the molecular level. Small-scale scalar mixing is important in a number of situations, e.g. turbulent combustion and environmental pollution (Namazian, Schefer \& Kelly 1988; Peters 2000; Warhaft 2000). A better understanding of the mean scalar dissipation rate $\overline{\varepsilon_{\theta}}$ is essential for optimizing the mixing of the scalar contaminants. It is defined as

$$
\overline{\varepsilon_{\theta}}=\kappa \overline{\left(\frac{\partial \theta}{\partial x_{i}}\right)\left(\frac{\partial \theta}{\partial x_{i}}\right)},
$$

$\dagger$ Email address for correspondence: habe@chofu.jaxa.jp 
where $\theta$ denotes the temperature fluctuation; $\kappa$ is the thermal diffusivity; the overbar denotes an averaged value; $x_{1}, x_{2}, x_{3}$ represent the streamwise, wall-normal and spanwise directions, respectively, and are used interchangeably with $x, y$ and $z$. The summation rule is applied to $i, j$ and $k$ but not to Greek suffices. Unfortunately, reliable information that is available for the scalar dissipation rate is rather limited especially near walls, where the measurements of $\overline{\varepsilon_{\theta}}$ (e.g. Sreenivasan, Antonia \& Danh 1977; Krishnamoorthy \& Antonia 1987) become difficult due to the small scales being affected by the mean and turbulent strain rates as well as the presence of the wall.

Recently, direct numerical simulations (DNSs) have become an essential tool for examining turbulence phenomena such as for assessing turbulence models, identifying different types of structures and quantifying the role these play in the context of the dynamics (e.g. Moin \& Mahesh 1998). For a non-isothermal turbulent channel flow, DNS was first performed by Kim \& Moin (1989) for $R e_{\tau}=u_{\tau} \delta / \nu=180$ (where $u_{\tau}$ is the friction velocity, $v$ the kinematic viscosity and $\delta$ the channel half-width) with uniform internal heating at $\operatorname{Pr}=0.1,0.71$ and 2.0. Subsequently, several DNS studies were carried out, also for a channel flow, with different thermal boundary conditions and different Reynolds and Prandtl numbers (e.g. Kasagi, Tomita \& Kuroda 1992; Kawamura, Abe \& Shingai 2000). In all the previously mentioned studies, the focus was mainly on large-scale transport.

DNSs for box turbulence (e.g. Ashurst et al. 1987; Ruetsch \& Maxey 1991, 1992; Holzer \& Siggia 1994; Pumir 1994; Vedula, Yeung \& Fox 2001; Brethouwer, Hunt \& Nieuwstadt 2003; Antonia \& Orlandi 2004) have provided valuable information on the small-scale features of this simple flow especially on the correlation between smallscale velocity and scalar fluctuations (e.g. the review by Antonia \& Orlandi 2003). On the other hand, quite a few DNS studies were performed on the small scales in a turbulent channel flow especially for the scalar dissipation rate (Kasagi et al. 1992; Antonia \& Kim 1994b; Johansson \& Wikström 1999; Orlandi, Leonardi \& Antonia 2006), which often encounter the low-Reynolds-number effects (Antonia \& Kim $1994 a$ ). To date, there has been no attempt to investigate the small-scale scalar mixing in the context of the organization of the flow. In particular, the relationship between the streaks, quasi-streamwise vortices and large-scale shear layers (e.g. Robinson 1991) and the scalar dissipation rate is yet to be examined in detail. It is well established that the quasi-streamwise vortices play a major role in maintaining the streaks and sustaining the turbulence in the vicinity of the wall. One may, therefore, expect that these vortices will influence the small-scale mixing significantly in this region.

In the present study, we carry out DNSs of a turbulent channel flow with passive scalar transport for a constant time-averaged heat-flux boundary condition at $\operatorname{Pr}=0.71$ and 3 values of $\operatorname{Re}_{\tau}(180,395$ and 640). The main objective is to study the relationship between the scalar dissipation field and the vorticity field. Since the fluctuating vorticity vector $\omega_{i}(i=1,2$ and 3 represent the streamwise, wall-normal and spanwise directions, respectively) and scalar derivative vector $\theta,{ }_{i}\left(\equiv \partial \theta / \partial x_{i}\right)$ give significant weighting to the small scales and given that the transport equations for the mean enstrophy $\left(\overline{\omega_{i} \omega_{i}}\right)$ and mean square scalar gradient $\left(\overline{\theta,{ }_{i} \theta,{ }_{i}}\right)$ are analogous in form (Corrsin 1953), we attempt to quantify the correlation between $\omega_{i}$ and $\theta,{ }_{i}$ as well as that between $\omega_{i} \omega_{i}$ and $\theta,{ }_{i} \theta,{ }_{i}$. We also consider the Reynolds number dependence of this correlation.

The high correlation between $u_{1}$ and $\theta$ in the near-wall region (e.g. Iritani, Kasagi \& Hirata 1985; Antonia, Krishnamoorthy \& Fulachier 1988; Kim \& Moin 1989; Kasagi et al. 1992; Kasagi \& Ohtsubo 1993) and its association with the velocity and thermal 
streaks (e.g. Kim 1988; Kim \& Moin 1989) implies a high correlation between $u_{1,2}\left(\simeq-\omega_{3}\right)$ and $\theta, 2$ as well as between $u_{1,3}\left(\simeq \omega_{2}\right)$ and $\theta, 3$ in this region. (The streamwise, wall-normal and spanwise velocity fluctuations are respectively denoted by $u_{1}, u_{2}$ and $u_{3}$; throughout this paper, $u, v, w$ are sometimes used interchangeably with $u_{1}, u_{2}, u_{3}$.) A special effort is therefore made to quantify the correlations between $\omega_{3}$ and $\theta, 2$ and between $\omega_{2}$ and $\theta,,_{3}$ and examine these in conjunction with instantaneous contours of these quantities in $x-z, x-y$ and $y-z$ planes. In particular, the association with the near-wall organized structures (viz. the streaks, internal shear layers and quasi-streamwise vortices) and the outer layer structures (viz. backs or fronts of large-scale $u_{1}$ and $\theta$ structures) is considered in some detail.

We also try to address the effects of the mean velocity and scalar gradients on the small-scale scalar field and those of vorticity on small-scale mixing, since unlike homogeneous isotropic turbulence, magnitudes of the mean velocity and scalar gradients are large near the wall.

This paper is organized as follows: The numerical methodology is described in $\S 2$. In $\S 3$, mean square values of the vorticity components and scalar derivatives are compared, and the degree of isotropy of $\omega_{i}$ and $\theta,{ }_{i}$ is also addressed. In $\S 4$, transport equations of $\overline{\omega_{i} \omega_{i}}$ and $\overline{\theta,{ }_{i} \theta,{ }_{i}}$ are examined to clarify the relationship between them especially with regard to the processes involved in the production and destruction of these quantities. The alignment between $\omega_{i}$ and $\theta,{ }_{i}$ is also investigated. The correlation coefficients between the vorticity and scalar derivative vectors are discussed in $\S 5$ in the context of the organized motions. Section 6 examines the topology of the scalar dissipation rate (note that the square of the instantaneous scalar gradient, $\theta,,_{i} \theta,{ }_{i}$, differs from the instantaneous scalar dissipation rate, $\kappa\left(\theta,{ }_{i} \theta,{ }_{i}\right)$, only by the constant factor $\kappa)$ and its relationship with that of the enstrophy, the energy dissipation rate and the vortical structures. In $\S \S 3-6$, the discussion covers both the inner (including viscous, buffer and also near-wall) and outer regions of the flow, and differences between these two flow regions in terms of the statistics and topologies associated with $\omega_{i}$ and $\theta,{ }_{i}$ are highlighted. We should note that the separation between the inner and outer regions is small at $R e_{\tau}=180$ and 395 but moderate at $R e_{\tau}=640$. A few checks of the computational accuracy are given in the Appendix.

\section{Numerical methodology}

The governing equations for the velocity and scalar fields are the incompressible three-dimensional continuity, Navier-Stokes and energy equations. A fully developed turbulent channel flow is driven by a constant streamwise mean pressure gradient. A passive scalar (temperature) is introduced by applying a constant time-averaged heat flux at each wall. All the variables computed from the Navier-Stokes and energy equations are normalized by the friction velocity $u_{\tau}$, the friction temperature $T_{\tau}\left(=Q_{w} / \rho c_{p} u_{\tau}\right)$ and the channel half-width $\delta$, where $Q_{w}$ is the averaged wall heat flux, $\rho$ the density and $c_{p}$ the specific heat at constant pressure. The statistical data are averaged in both space ( $x$ and $z$ directions) and time $(t)$.

For the energy equation, an instantaneous temperature difference $\Theta$ defined by

$$
T=\frac{\partial\left\langle T_{w}\right\rangle}{\partial x} x-\Theta
$$

(where $T$ and $T_{w}$ are the local temperature and wall temperature, respectively, and the angular brackets represent integration over $z$ and $t$ ) is used since $\langle T\rangle,\left\langle T_{w}\right\rangle,\left\langle T_{m}\right\rangle$ increase linearly in the $x$ direction for the present configuration (with $\left\langle T_{m}\right\rangle$ denoting 
the bulk mean temperature). The introduction of $\Theta$ enables the assumption that the temperature fluctuation is zero at the wall, viz. the no slip boundary condition applies in the $y$ direction. For the other $(x$ and $z$ ) directions, periodic boundary conditions are imposed. The first term on the right-hand side of (2.1) can be written as

$$
\frac{\partial\left\langle T_{m}^{+}\right\rangle}{\partial x^{\#}}=\frac{\partial\left\langle T_{w}^{+}\right\rangle}{\partial x^{\#}}=\frac{2}{\int_{0}^{2} \bar{U}_{1} d y^{\#}}
$$

(see also Kasagi et al. 1992; Kawamura et al. 1998), where the superscripts + and \# denote normalizations by wall variables $\left(v / u_{\tau}\right.$ and $\left.u_{\tau}\right)$ and the channel half-width $(\delta)$, respectively. Using (2.1) and (2.2), the energy equation can be expressed as

$$
\frac{\partial \Theta^{+}}{\partial t^{\#}}+U_{j}^{+} \frac{\partial \Theta^{+}}{\partial x_{j}^{\#}}=\frac{1}{R e_{\tau} \cdot \operatorname{Pr}} \frac{\partial^{2} \Theta^{+}}{\partial x_{j}^{\# 2}}+U_{1}^{+} \frac{2}{\int_{0}^{2} \bar{U}_{1} d y^{\#}},
$$

where $U_{i}$ is the instantaneous velocity in the $i$ th direction. Note that the two walls are kept cold and act to cool down the hot fluid (Kasagi et al. 1992; Kawamura et al. 1998; Kawamura, Abe \& Matsuo 1999), which contrasts with the more common experimental situation in which the wall is heated (e.g. Krishnamoorthy \& Antonia 1987).

A fractional step method is adopted with a semi-implicit time-advancement scheme. For the viscous terms with wall-normal derivatives, the Crank-Nicolson method is used. For the other terms, the low-storage third-order Runge-Kutta method (Spalart, Moser \& Rogers 1991) is employed. For the spatial discretization, the finite-difference method is used. The fourth-order central scheme (Morinishi et al. 1998) is applied in the $x$ and $z$ directions, whereas the second-order one is applied in the $y$ direction. Further details of the numerical methodology can be found in Abe, Kawamura \& Matsuo (2001, 2004b) and Antonia, Abe \& Kawamura (2008).

For the spatial discretization, most of the existing DNS studies of the scalar dissipation rate employ the spectral method (e.g. Antonia \& Kim 1994b), while the present study uses the finite-difference method (Abe et al. 2001, 2004b; Abe, Kawamura \& Choi 2004a). The latter method is generally inferior to the spectral method in terms of the numerical accuracy when one adopts the same number of grid (or mode) points (e.g. Kravchenko \& Moin 1997). To overcome this difficulty, the present numerical scheme has higher order accuracy in the $x$ and $z$ directions, and a large number of grid points are used in all three directions to resolve the Kolmogorov and Batchelor length scales (viz. $\eta\left(\equiv\left(v^{3} / \bar{\varepsilon}\right)^{1 / 4}\right)$ and $\eta_{B}\left(\equiv \eta P r^{-1 / 2}\right)$, where $\bar{\varepsilon}$ is the mean energy dissipation rate defined by $\left.\bar{\varepsilon} \equiv v \overline{\left(u_{i, j}\left(u_{i, j}+u_{j, i}\right)\right)}\right)$ as accurately as possible. We should mention that for the finite-difference method, the high wavenumber part of the spectrum does not suffer from the energy pileup that has been observed with the pseudo-spectral approach (see, for example, Antonia \& Orlandi 2004).

The computational domain size $\left(L_{x} \times L_{y} \times L_{z}\right)$, number of grid points $\left(N_{x} \times N_{y} \times N_{z}\right)$, spatial resolution $(\Delta x, \Delta y, \Delta z)$ and the sampling duration $\left(t_{\text {sam }}\right)$ are given in table 1, where the superscript * denotes normalization by Kolmogorov scales $\left(\eta\right.$ and $\left.v_{K}\right)$ and the subscript $c$ represents the centreline value $\left(v_{K}\left(\equiv(\nu \bar{\varepsilon})^{1 / 4}\right)\right.$ denotes the Kolmogorov velocity scale). The spatial resolution at the centreline is smaller than about $0.8 \eta$ in the $x$ and $z$ directions and about $1.6 \eta$ in the $y$ direction for all the cases examined. Since $\operatorname{Pr}$ is smaller than 1 , the Batchelor scale $\eta_{B}$ is slightly larger than the Kolmogorov scale. Also, included in table 1 is the Taylor microscale 


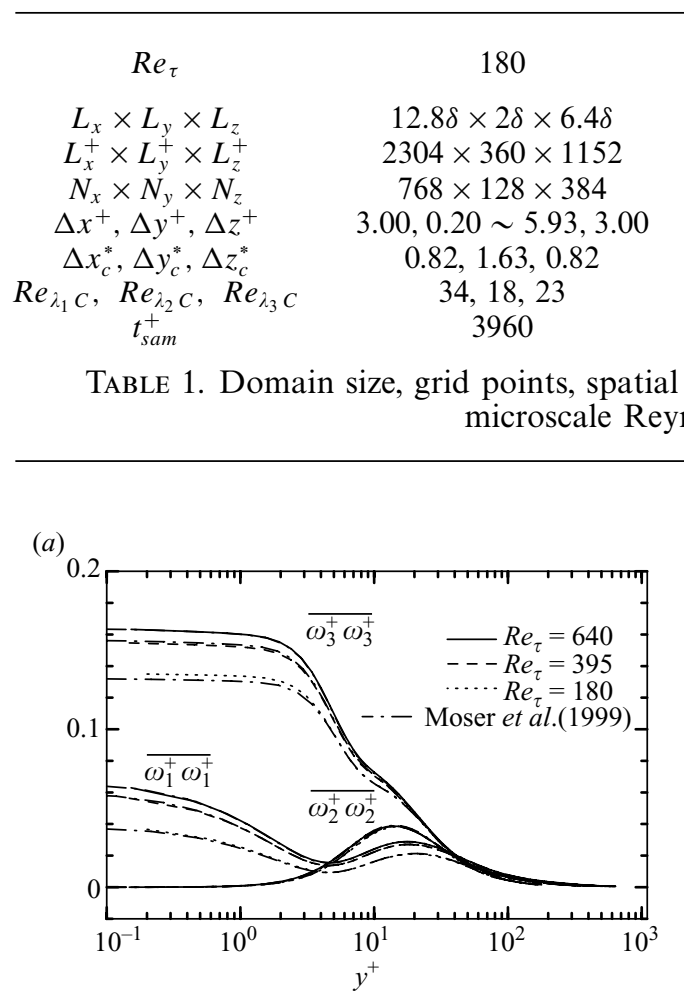

395
$12.8 \delta \times 2 \delta \times 6.4 \delta$
$5056 \times 790 \times 2528$
$1536 \times 192 \times 768$
$3.29,0.15 \sim 6.52,3.29$
$0.74,1.47,0.74$
$51,30,35$
5008

640

$12.8 \delta \times 2 \delta \times 6.4 \delta$

$8192 \times 1280 \times 4096$

$2048 \times 256 \times 1024$

$4.00,0.15 \sim 8.02,4.00$

$0.82,1.64,0.82$

$66,40,46$

6385

TABLE 1. Domain size, grid points, spatial resolution, sampling duration and Taylor microscale Reynolds numbers.

FIGURE 1. Mean square values of the vorticity components and temperature derivatives normalized by wall units: (a) $\overline{\omega_{\alpha}^{+} \omega_{\alpha}^{+}}$; $(b) \overline{\theta,{ }_{\alpha}^{+} \theta,{ }_{\alpha}^{+}}$. For $\overline{\omega_{\alpha}^{+} \omega_{\alpha}^{+}}$, the Reynolds numbers of Moser et al. (1999) are $R e_{\tau}=180,395$ and 590. For $\frac{\alpha,{ }_{\alpha}^{+} \theta,{ }_{\alpha}^{+}}{,}, R e_{\tau}=180$ for Antonia \& Kim (1994b) and 150 for Kasagi et al. (1992); the studies employed different thermal boundary conditions, viz. a constant internal heat source and a constant wall heat-heat flux, respectively. In this and subsequent figures the Prandtl number, $\mathrm{Pr}$, is equal to 0.71 .

Reynolds number at the centreline defined by

$$
\operatorname{Re}_{\lambda_{i} C}=\frac{u^{\prime} \lambda_{i}}{v}
$$

where $\lambda_{i}^{+} \equiv\left(\overline{u_{1}^{+2}} / \overline{u_{1, i}^{+2}}\right)^{1 / 2}$ is the Taylor microscale; the prime denotes a root mean square (r.m.s.) value; and the subscript $\mathrm{C}$ refers to the centreline. The present magnitudes of $R e_{\lambda_{i} C}$ are smaller than for DNS box turbulence data with a passive scalar (Vedula et al. 2001; Watanabe \& Gotoh 2004, 2007; Yeung, Donzis \& Sreenivasan 2005). A few validation checks of the computational accuracy are given in the Appendix.

\section{Distributions of $\overline{\omega_{\alpha} \omega_{\alpha}}$ and $\overline{\theta,{ }_{\alpha} \theta, \alpha}-$ comparison with isotropy}

In this section, we consider the magnitudes of $\overline{\omega_{\alpha} \omega_{\alpha}}$ and $\overline{\theta, \alpha \theta,_{\alpha}}$ and how the mean velocity and temperature gradients affect their departure from isotropy.

Figure 1 shows the components of $\overline{\omega_{\alpha} \omega_{\alpha}}$ and $\overline{\theta,_{\alpha} \theta_{, \alpha}}$ normalized by wall variables. The DNS data of Moser, Kim \& Mansour (1999) at $R e_{\tau}=180,395$ and 590 for $\overline{\omega_{\alpha} \omega_{\alpha}}$ and those of Antonia \& $\operatorname{Kim}\left(1994 b ; e_{\tau}=180\right.$ and $\left.P r=0.71\right)$ and Kasagi et al. (1992; $\operatorname{Re}_{\tau}=150$ and $\left.P r=0.71\right)$ for $\overline{\theta_{, \alpha} \theta_{, \alpha}}$ are included in figures $1(a)$ and $1(b)$, respectively. The agreement between the present distributions and those of Kasagi 
et al. (1992) and Moser et al. (1999) is satisfactory. The relatively poor agreement with Antonia \& Kim (1994b) may be due to the different thermal boundary condition used by them. We note that $\overline{\omega_{3}^{+} \omega_{3}^{+}}$and $\overline{\omega_{2}^{+} \omega_{2}^{+}}$exhibit nearly the same behaviour as $\overline{\theta,{ }_{2}^{+} \theta,,_{2}^{+}}$and $\overline{\theta,{ }_{3}^{+} \theta,,_{3}^{+}}$, respectively, whereas $\overline{\omega_{1}^{+} \omega_{1}^{+}}$behaves in a manner different to that of $\overline{\theta,{ }_{1}^{+} \theta,{ }_{1}^{+}}$. This latter difference may reflect the difference between the solenoidal nature of the vorticity vector $\left(\nabla \cdot \omega_{i} \equiv 0\right)$ and the lamellar nature of the scalar derivative vector $\left(\nabla \times \theta,{ }_{i} \equiv 0\right)$, as first indicated by Corrsin (1953) when comparing the transport equations of $\overline{\theta,{ }_{i} \theta, i}$ and $\overline{\omega_{i} \omega_{i}}$. In this context, there is evidence to suggest that the near-wall distributions of $\overline{\theta, 2}, \overline{\theta, 2}, \overline{\theta, 2}$ are quite similar to those of $\overline{u_{1,1}^{2}}, \overline{u_{1,2}^{2}}, \overline{u_{1,3}^{2}}$ even when $\operatorname{Pr}=0.71$ (Antonia, Kim \& Browne 1991). Since $\omega_{2} \approx u_{1,3}$ and $\omega_{3} \approx-u_{1,2}$ near the wall, the similarity between $\overline{\omega_{2}^{+} \omega_{2}^{+}}$and $\overline{\theta,{ }_{3}^{+} \theta,{ }_{3}^{+}}$and between $\overline{\omega_{3}^{+} \omega_{3}^{+}}$and $\overline{\theta,{ }_{2}^{+} \theta,,_{2}^{+}}$ arises from the strong correlation between $u_{1}$ and $\theta$ (Kim \& Moin 1989; Antonia \& Kim 1991). The dissimilarity between $\overline{\omega_{1}^{+} \omega_{1}^{+}}$and $\overline{\theta,{ }_{1}^{+} \theta,{ }_{1}^{+}}$is most likely related to the different natures of the motions which contribute to $\omega_{1}$ and $\theta,,_{1}$ (quasi-streamwise vortices versus internal shear/thermal layers).

In the near-wall region, the largest contributions to the enstrophy $\left(\overline{\omega_{i}^{+} \omega_{i}^{+}}\right)$and scalar dissipation rate $\left(\kappa\left(\overline{\theta,{ }_{i}^{+} \theta,{ }_{i}^{+}}\right)\right)$are from $\overline{\omega_{3}^{+} \omega_{3}^{+}}$and $\overline{\theta,{ }_{2}^{+} \theta,{ }_{2}^{+}}$, respectively. The magnitudes

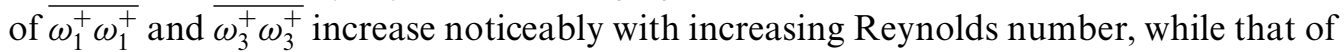
$\overline{\omega_{2}^{+} \omega_{2}^{+}}$does not change appreciably with the Reynolds number (figure $1 a$ ). The rate of increase for $\overline{\omega_{1}^{+} \omega_{1}^{+}}$and $\overline{\omega_{3}^{+} \omega_{3}^{+}}$is significant between $R e_{\tau}=180$ and $R e_{\tau}=395$ but only moderate between $R e_{\tau}=395$ and $R e_{\tau}=640$. The magnitude of $\overline{\theta,{ }_{2}^{+} \theta,{ }_{2}^{+}}$increases significantly as $R e_{\tau}$ increases, whereas the magnitudes of $\overline{\theta_{1}^{+} \theta_{1}^{+}}$and $\overline{\theta,{ }_{3}^{+} \theta,{ }_{3}^{+}}$remain unchanged (figure $1 b$ ). The near-wall dependence on $R e_{\tau}$ of the scalar dissipation rate thus comes substantially from $\overline{\theta,{ }_{2}^{+} \theta,,_{2}^{+}}$. The significant increase in $\overline{\omega_{1}^{+} \omega_{1}^{+}}, \overline{\omega_{3}^{+} \omega_{3}^{+}}$and $\overline{\theta,{ }_{2}^{+} \theta,{ }_{2}^{+}}$occurs for $y^{+}<3$ and is essentially caused by the straining induced by the wall (note that $\overline{\omega_{1}^{+} \omega_{1}^{+}} \approx \overline{u_{3,2}^{+} u_{3,2}^{+}}$and $\overline{\omega_{3}^{+} \omega_{3}^{+}} \approx \overline{u_{1,2}^{+} u_{1,2}^{+}}$in the vicinity of the wall). On the other hand, the approximate independence on $R e_{\tau}$ of $\overline{\omega_{2}^{+} \omega_{2}^{+}}$and $\overline{\theta,_{3}^{+} \theta,_{3}^{+}}$seems consistent with the independence on $R e_{\tau}$ of the spanwise streak spacing (Smith $\&$ Metzler 1983), after normalization by wall variables. The approximate independence on $R e_{\tau}$ of $\overline{\theta,{ }_{1}^{+} \theta,{ }_{1}^{+}}$may be associated with approximate independence of the streamwise separation normalized by wall variables between internal shear and thermal layers (Kim 1988; Kim \& Moin 1989).

In figure 1 , the magnitudes, for each value of $\alpha$, of either $\overline{\omega_{\alpha} \omega_{\alpha}}$ or $\overline{\theta_{, \alpha} \theta,_{\alpha}}$ are nearly equal in the outer layer, which may indicate that the vorticity and scalar dissipation fields approach isotropy as the channel centreline is approached. To examine how they approach isotropy, the mean square values of $\overline{\omega_{\alpha} \omega_{\alpha}}$ and $\overline{\theta, \alpha \theta,_{\alpha}}$ for $R e_{\tau}=180$, 395 and 640 at $\operatorname{Pr}=0.71$ are normalized by Kolmogorov and Batchelor scales $\left(v_{K}\right.$, $\left.\theta_{B}, \eta\right)$ and are shown in figure $2\left(\theta_{B}\left(\equiv\left(\bar{\varepsilon}_{\theta} \nu^{1 / 2} \bar{\varepsilon}^{-1 / 2}\right)^{1 / 2}\right)\right.$ is the Batchelor temperature scale). Note that $\overline{\omega_{\alpha}^{*} \omega_{\alpha}^{*}}$ denotes normalization by $\left(v_{K} / \eta\right)^{2}$ or equivalently $\left(1 / t_{K}\right)^{2}$ ( $\equiv \bar{\varepsilon} / v$, where $t_{K}$ is the Kolmogorov time scale), whilst $\overline{\theta,{ }_{\alpha}^{*} \theta,{ }_{\alpha}^{*}}$ denotes normalization by $\left(\theta_{B} / \eta\right)^{2}$ or equivalently $\left(\overline{\theta,{ }_{i} \theta, i} / \operatorname{Pr}\right)\left(\equiv \overline{\varepsilon_{\theta}} / \nu\right)$. For local isotropy, $\overline{\omega_{\alpha}^{*} \omega_{\alpha}^{*}}$ and $\overline{\theta,{ }_{\alpha}^{*} \theta,{ }_{\alpha}^{*}}$ should be equal to $1 / 3$ and $\operatorname{Pr} / 3$, respectively. In figure 2, the departure from

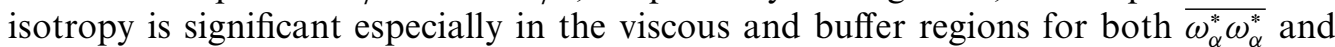

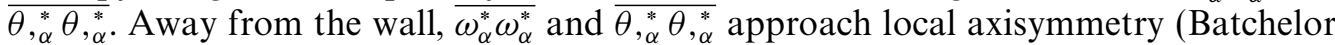
1946; Chandrasekhar 1950; George \& Hussein 1991) rather than local isotropy for

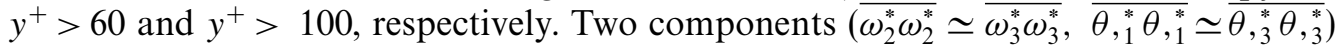
are nearly equal, and their magnitudes are close to isotropy, while the remaining 
(a)

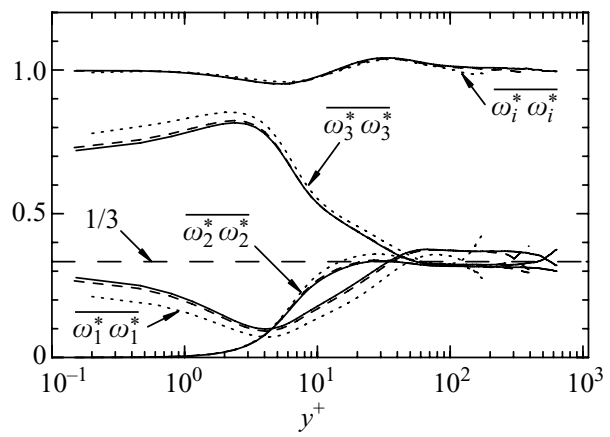

(b)

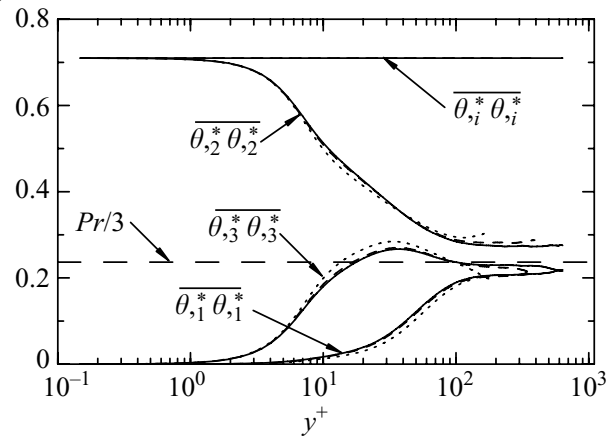

FiguRE 2. Mean square values of the vorticity components and temperature derivatives for

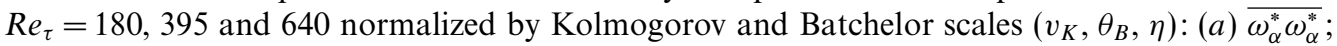
(b) $\overline{\theta,{ }_{\alpha}^{*} \theta,{ }_{\alpha}^{*}} ;-, R e_{\tau}=640 ;-\cdots .-, R e_{\tau}=395 ; \cdots \cdots \cdots, R e_{\tau}=180$.

component $\left(\overline{\omega_{1}^{*} \omega_{1}^{*}}, \overline{\theta,{ }_{2}^{*} \theta,{ }_{2}^{*}}\right)$ is larger than the other two, thus exhibiting the largest departure from isotropy. The anisotropy is more enhanced for $\overline{\theta,{ }_{\alpha}^{*} \theta,_{\alpha}^{*}}$ than $\overline{\omega_{\alpha}^{*} \omega_{\alpha}^{*}}$, consistent with the general expectation that the scalar dissipation rate is more anisotropic than the vorticity (e.g. Sreenivasan 1991; Antonia \& Kim 1994b). In the region $y^{+}>100$ and $y / \delta<0.7$, where the departure from isotropy remains

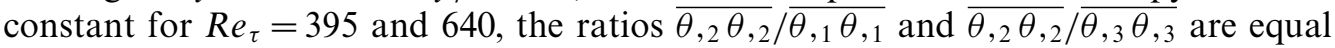
to about $1.3 \sim 1.6$ and $1.2 \sim 1.3$, respectively. In contrast to isotropic turbulence with a superimposed mean temperature gradient (e.g. the review of Antonia \& Orlandi 2003), little information is available for turbulent shear flows. For example, Sreenivasan et al.

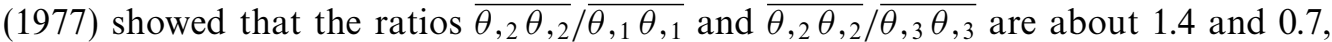
respectively, in a turbulent boundary layer. Tavoularis \& Corrsin (1981) reported a value of 1.82 for the ratio of $\overline{\theta,{ }_{\alpha} \theta, \alpha}$ between directions parallel and perpendicular to the imposed mean scalar gradient in a nearly homogeneous turbulent shear flow. For a turbulent boundary layer, Krishnamoorthy \& Antonia (1987) found almost the same trend as Sreenivasan et al. (1977), e.g. $\overline{\theta,,_{2} \theta, 2} / \overline{\theta,,_{1} \theta, 1}=1.4, \overline{\theta,,_{2} \theta, 2} / \overline{\theta,,_{3} \theta,,_{3}}=0.9$, at $y / \delta=0.25$ (here $\delta$ denotes the boundary layer thickness). The present data at $R e_{\tau}=640$ indicate that $\overline{\theta,,_{2} \theta, 2} / \overline{\theta,,_{1} \theta, 1}=1.4$ and $\overline{\theta, 2 \theta} / \overline{\theta,,_{3} \theta,,_{3}}=1.2$. Speculatively, the poor agreement for $\overline{\theta, 2 \theta, 2} / \overline{\theta,,_{3} \theta, 3}$ may reflect a difference in outer layer properties between the channel and the boundary layer. From the above considerations, one may infer that the levels of anisotropy in $\overline{\omega_{\alpha} \omega_{\alpha}}$ and $\overline{\theta,_{\alpha} \theta,_{\alpha}}$ may be associated with the mean velocity and temperature gradients.

It seems natural to inquire whether $\overline{\omega_{\alpha}^{*} \omega_{\alpha}^{*}}$ and $\overline{\theta,{ }_{\alpha}^{*} \theta,_{\alpha}^{*}}$ approach isotropy at the centre of the channel due to the disappearance of the mean velocity and temperature gradients there. In this context, Antonia \& Mi (1993) found that on the axis of a round jet, the three components of the temperature dissipation rate are approximately equal. They also indicated that this behaviour differs from that in a plane jet (Antonia \& Browne 1983) or a wake (Browne, Antonia \& Shah 1987). The behaviour in the two latter flows seems to correspond more closely to that of the present data. The previous observations imply that the departure from isotropy of mean square derivative values may be flow dependent. In a turbulent channel flow, the transport of turbulent energy and scalar variance across the centreline by the large-scale motion is likely to cause departures from local isotropy. 
In figure 2 , the sum of the mean square vorticity components, $\overline{\omega_{i}^{*} \omega_{i}^{*}}$, should be equal to 1 provided local homogeneity is satisfied, viz. $\bar{\varepsilon}=v \overline{\omega_{i} \omega_{i}}$. However, local homogeneity is not strictly satisfied for $\overline{\omega_{i}^{*} \omega_{i}^{*}}$ near the wall (figure $2 a$ ). Such a departure from homogeneity was reported for the energy dissipation rate by Antonia et al. (1991). Since the general relation between the energy dissipation rate and enstrophy is given by $\bar{\varepsilon}=v\left(\overline{\omega_{i} \omega_{i}}+2 \overline{u_{i, j} u_{i, j}}\right)$, the departure from homogeneity is due to the term $v\left(2 \overline{u_{i, j} u_{j, i}}\right)$ which is closely associated with vortical motions, such as the near-wall streamwise vortices (e.g. Robinson 1991).

\section{Transport equations of $\overline{\omega_{i} \omega_{i}}$ and $\overline{\theta_{, i} \theta_{, i}}$}

Corrsin (1953) first drew attention to the analogous form of the transport equations for the mean enstrophy, $\overline{\omega_{i} \omega_{i}}$, and the mean square scalar gradient, $\overline{\theta,{ }_{i} \theta, i}$, notwithstanding the different natures of these two quantities. This suggests a close association between vorticity and scalar dissipation rate fields. With the use of DNS databases, the topic has been pursued with vigour for homogeneous isotropic turbulence (e.g. Ashurst et al. 1987; Ruetsch \& Maxey 1991, 1992; Pumir 1994) and homogeneous sheared turbulence (e.g. Nomura \& Elghobashi 1992). In wall turbulence, however, the relationship between the two transport equations is still unclear, especially near the wall, where the mean strain rate is large, and the quasi-streamwise vortices play an important role in sustaining turbulence. Such an investigation should help in terms of constructing more reliable models for $\overline{\varepsilon_{\theta}}$ in near-wall flows.

For the present configuration, the transport equation of $\overline{\omega_{i} \omega_{i}}$ normalized by wall units may be written

$$
\begin{aligned}
0= & \underbrace{-2 \overline{\omega_{i}^{+} u_{j}^{+}} \frac{\partial \bar{\Omega}_{i}^{+}}{\partial x_{j}^{+}}}_{1}+\overline{\omega_{i}^{+}\left(\frac{\partial u_{j}^{+}}{\partial x_{i}^{+}}+\frac{\partial u_{i}^{+}}{\partial x_{j}^{+}}\right)} \bar{\Omega}_{j}^{+}+\underbrace{\overline{\omega_{i}^{+} \omega_{j}^{+}}\left(\frac{\partial \bar{U}_{i}^{+}}{\partial x_{j}^{+}}+\frac{\partial \bar{U}_{j}^{+}}{\partial x_{i}^{+}}\right)}_{3} \\
& +\underbrace{\overline{\omega_{i}^{+} \omega_{j}^{+}\left(\frac{\partial u_{i}^{+}}{\partial x_{j}^{+}}+\frac{\partial u_{j}^{+}}{\partial x_{i}^{+}}\right)}}_{5}-\underbrace{\frac{\partial}{\partial x_{j}^{+}}\left(\overline{\omega_{i}^{+} \omega_{i}^{+} u_{j}^{+}}\right)}_{6}+\underbrace{\frac{\partial^{2}}{\partial x_{j}^{+} \partial x_{j}^{+}}\left(\overline{\omega_{i}^{+} \omega_{i}^{+}}\right)}_{5} \\
& -\underbrace{2}_{\left.\frac{\partial \omega_{i}^{+}}{\partial x_{j}^{+}}\right)\left(\frac{\partial \omega_{i}^{+}}{\partial x_{j}^{+}}\right)},
\end{aligned}
$$

where $\Omega_{i}$ is the instantaneous vorticity in the $i$ th direction. Also, the transport equation of $\overline{\theta,{ }_{i} \theta,{ }_{i}}$ normalized by wall units is given by

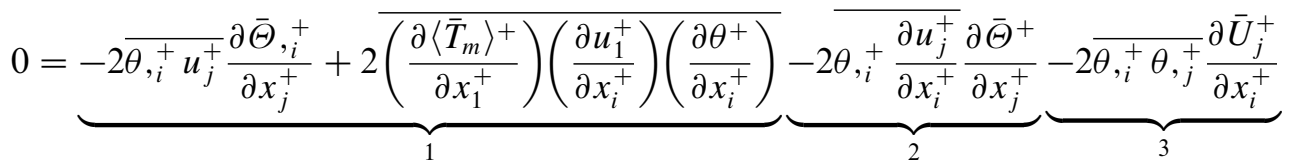

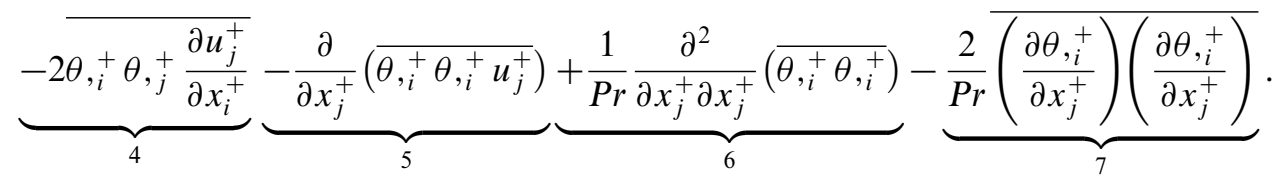


(a)

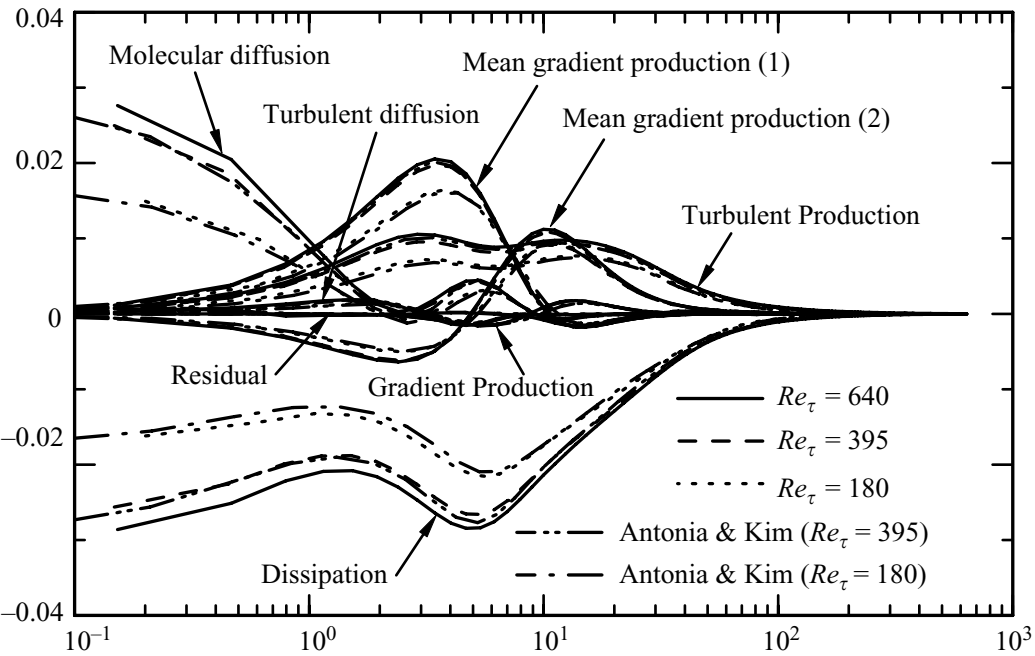

(b)

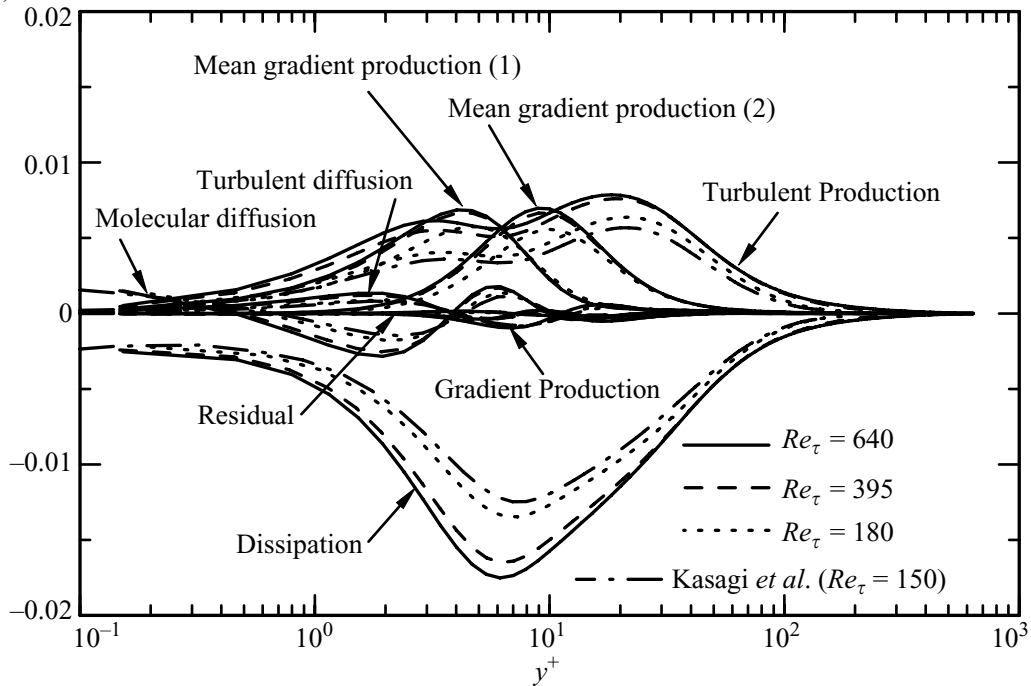

FIGURE 3. Budgets of $\overline{\omega_{i} \omega_{i}}$ and $\overline{\theta,,_{i} \theta,{ }_{i}}$ normalized by wall units: $(a) \overline{\omega_{i}^{+} \omega_{i}^{+}} ;(b) \overline{\theta,_{i}^{+} \theta,_{i}^{+}}$. For $\overline{\omega_{i}^{+} \omega_{i}^{+}},-\cdot \cdot \cdot \cdot$, Antonia \& Kim $(1994 a)$ at $R e_{\tau}=180 ;-\cdots-\cdots-$, Antonia \& Kim (1994a) at $R e_{\tau}=395$. For $\overline{\theta,_{i}^{+} \theta,_{i}^{+}},-\cdot-\cdot-$. Kasagi et al. (1992) at $R e_{\tau}=150$.

Note that in (4.1) and (4.2), the terms 1, 2, 3, 4 denote the gradient production, mean gradient production (1), mean gradient production (2) and turbulent production, respectively, whereas the terms 5, 6, 7 represent the turbulent diffusion, molecular diffusion and dissipation, respectively. The budgets of $\overline{\omega_{i} \omega_{i}}$ and $\overline{\theta,{ }_{i} \theta,{ }_{i}}$, normalized by wall variables, are shown in figure 3 , where a comparison with previous DNS data (Kasagi et al. 1992; Antonia \& Kim 1994a) is made. For a turbulent channel flow, in contrast to isotropic turbulence (e.g. Ruetsch \& Maxey 1992; Vedula et al. 2001), the mean gradient terms cannot be neglected but are of the same order of magnitude as the turbulent production term near the wall. As noted by Corrsin (1953), the similarity between the transport equations of $\overline{\omega_{i} \omega_{i}}$ and $\overline{\theta,{ }_{i} \theta,{ }_{i}}$ seems convincing, 
although differences can be discerned between the mean gradient production and turbulent production terms. The differences near the wall are due to the different boundary conditions. For the transport equations of both $\overline{\omega_{i} \omega_{i}}$ and $\overline{\theta,{ }_{i} \theta, i}$, Reynoldsnumber effects do not appear in the gradient production term but in the other three production terms, consistent with those for the transport equation of $\bar{\varepsilon}$ (Rodi \& Mansour 1993). The rate of increase from $R e_{\tau}=180$ to $R e_{\tau}=395$ is larger than that from $R e_{\tau}=395$ to $R e_{\tau}=640$. Tennekes \& Lumley (1972) provided order of magnitude estimates for each term in the transport equations of $\overline{\omega_{i} \omega_{i}}$. They suggested that the ratio of the mean gradient production to the difference between the turbulent production and the dissipation terms is of the order $1 / R e^{1 / 2}$. However, as can be seen in the budget of $\bar{\varepsilon}$ obtained by Rodi \& Mansour (1993), such Reynolds-number effects do not appear in the budgets of $\overline{\omega_{i} \omega_{i}}$ and $\overline{\theta_{, i} \theta,_{i}}$ away from the wall (not shown here), which may be useful for the development of turbulence models especially in the context of the transport equation for $\overline{\varepsilon_{\theta}}$ (e.g. Nagano \& Kim 1988; Yoshizawa 1988; Johansson \& Wikström 1999).

For each budget, a better understanding of the turbulent production terms is necessary in order to gain further insight into the production process in view of the leading-order magnitude of these terms across the channel (figure 3). The terms, which can be rewritten as $2 \overline{\omega_{i} \omega_{j} s_{i j}}$ (4.1) and $-2 \overline{\theta_{i} \theta{ }_{, j} s_{i j}}$ (4.2), have the fluctuating strain rate tensor $s_{i j}\left(\equiv(1 / 2)\left(u_{i, j}+u_{j, i}\right)\right)$ in common. The magnitudes of $2 \overline{\omega_{i} \omega_{j} s_{i j}}$ and $-2 \overline{\theta,{ }_{i} \theta,{ }_{j} s_{i j}}$ depend on the relative magnitudes and orientations of $\omega_{i}$ and $\theta,{ }_{i}$ and also $s_{i j}$; e.g. the extensional (positive) and compressive (negative) strain rates lead to

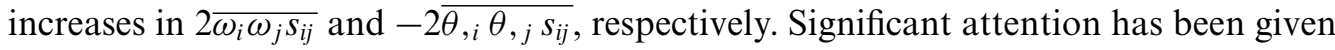
to the alignments of $\omega_{i}$ and $\theta, i$ with respect to the directions of the principal rates of strain in homogeneous isotropic turbulence (e.g. Ashurst et al. 1987; Ruetsch \& Maxey 1992; Pumir 1994) and homogeneous sheared turbulence (e.g. Nomura \& Elghobashi 1992). We investigate this issue further for the present flow. In particular, the emphasis is on the near-wall region. Probability density functions (p.d.f.s) for the cosine of the angle between the vorticity and the principal strain rate directions and also between the scalar gradient and the principal strain rate directions are given in figure 4 , where $a_{i}, b_{i}, c_{i}$ denote the eigenvectors corresponding to the directions of the eigenvalues $a, b, c$, respectively. Since $s_{i j}$ is the symmetric tensor, $a, b, c$ are real with $a+b+c=0$ (solenoidal condition) and $a \geqslant b \geqslant c(a \geqslant 0 \geqslant c)$. Note that subsequent results for the principal strain rates are obtained using one instantaneous realization as in Blackburn, Mansour \& Cantwell (1996). Near the wall, $\omega_{i}$ is aligned with the intermediate $(b)$ strain rate, consistent with the finding of Blackburn et al. (1996), who noted the correspondence between the $b$ strain rate and mean spanwise vorticity (viz. mean strain rate tensor) directions (for a possible kinematical explanation, see Jiménez 1992). On the other hand, the orientation of $\theta,{ }_{i}$ is at about $45^{\circ}$ to the most compressive $(c)$ and extensional $(a)$ strain rate directions $(\cos \theta=0.71)$. As in the case of $\omega_{i}, \theta,{ }_{i}$ is aligned with the direction of the mean strain rate tensor. The preferred orientation of $\theta, i$ to the mean strain rate was also reported by Nomura \& Elghobashi (1992) for homogeneous sheared turbulence. The present observations with regard to the alignment may readily be expected, since $\overline{\omega_{3} \omega_{3}}$ and $\overline{\theta_{, 2} \theta, 2}$ are the most dominant components near the wall (figure 1). At the channel centreline, $\omega_{i}$ and $\theta,_{i}$ tend to align with the intermediate $(b)$ and compressive $(c)$ strain rates, respectively. The distributions are almost the same as for isotropic turbulence (e.g. Ashurst et al. 1987; She, Jackson \& Orszag 1990; Ruetsch \& Maxey 1992; Pumir 1994; Vincent \& Meneguzzi 1994; Vedula et al. 2001; Brethouwer et al. 2003; see also Sreenivasan \& Antonia 1997). 
(a)

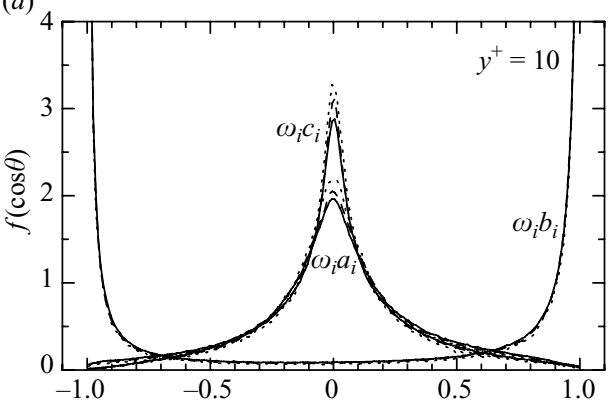

(c)

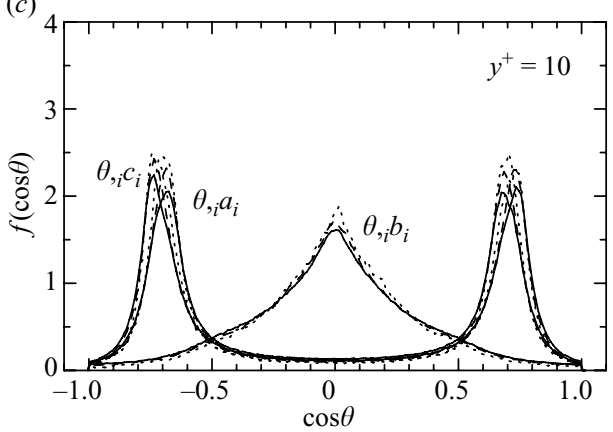

(b)

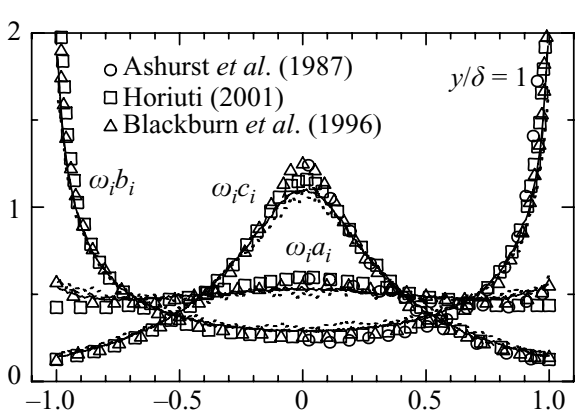

(d)

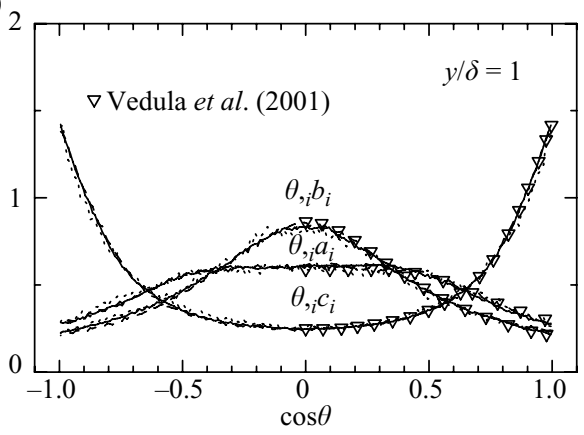

Figure 4. P.d.f.s of the cosine of the angle between the vorticity and the principal strain rate directions $((a)$ and $(b))$ and between the scalar gradient and the principal strain rate directions $((c)$ and $(d)):(a),(c) y^{+}=10 ;(b),(d) y / \delta=1 ;-, R e_{\tau}=640 ;-\cdots-.-R_{\tau}=395$; ........, $R e_{\tau}=180$; O, Ashurst et al. (1987) at $R_{\lambda}=87$ (isotropic turbulence); $\triangle$, Horiuti (2001) at $R_{\lambda}=87.6$ (isotropic turbulence); $\square$, Blackburn et al. (1996) for $\operatorname{Re}_{\tau}=395$ at $y / \delta=1$ (turbulent channel flow); $\nabla$, Vedula et al. (2001) at $R_{\lambda}=243$ (isotropic turbulence with a mean temperature gradient). Note that $R_{\lambda}$ denotes the Reynolds number based on the Taylor microscale and the r.m.s. of $u$.

The preferred orientation of $\omega_{i}$ and $\theta,_{i}$ is reflected in the mean square values projected along each of the principal strain eigenvectors (the distributions are not shown here). As inferred from figure 4 , the largest contributions to the mean square values of $\omega_{i}$ and $\theta,{ }_{i}$ are along the $b$ and $c$ strain rate directions, respectively, across the major portion of the channel. As the wall is approached, their magnitudes change gradually due to the variation of the mean strain rate. Near the wall, the contribution to $\overline{\omega_{i} \omega_{i}}$ is almost along the $b$ strain rate direction, whilst that to $\overline{\theta,{ }_{i} \theta,{ }_{i}}$ is at about $45^{\circ}$ to the $a$ and $c$ strain rate directions, suggesting a preference to align with the mean strain rate tensor. The same is true for structures of $\omega_{i} \omega_{i}$ and $\theta,{ }_{i} \theta,{ }_{i}$, although the instantaneous fields are not shown here (see also $\S 6$ ). In the near-wall region, $\omega_{i} \omega_{i}$ is mostly aligned with the $b$ strain rate direction, whereas the orientation of $\theta,{ }_{i} \theta,{ }_{i}$ lies, to a large degree, at about $45^{\circ}$ to the $a$ and $c$ strain rate directions. The quantities $\omega_{i} \omega_{i}$ and $\theta,{ }_{i} \theta,{ }_{i}$ are space filling (with momentum and thermal streaks $\left(\omega_{3}\right.$ and $\theta, 2)$ and quasi-streamwise vortices $\left(\omega_{1}\right)$ being the most dominant contributors) so that $\left(\omega_{i} b_{i}\right)^{2} / \omega_{i}^{2} \approx 1$ and $\left(\theta,{ }_{i} a_{i}\right)^{2} / \theta,{ }_{i} \simeq\left(\theta,{ }_{i} c_{i}\right)^{2} / \theta,{ }_{i} \approx 0.5$ hold over most of the wall region. In the outer region, they occur only sparsely. As for homogeneous isotropic turbulence, the intense regions of $\omega_{i} \omega_{i}$ and $\theta,{ }_{i} \theta,_{i}$ (e.g. annular regions of tubelike vortices $\left(\omega_{i} \omega_{i}\right)$ and sheetlike structures $\left.\left(\theta,{ }_{i} \theta, i\right)\right)$ are aligned with the $b$ and $c$ strain rate directions, respectively. 
(a)

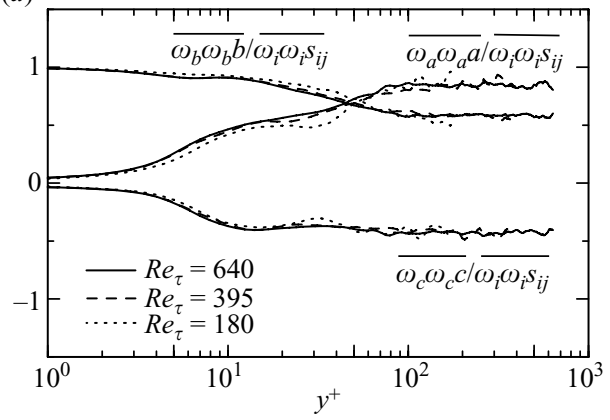

(b)

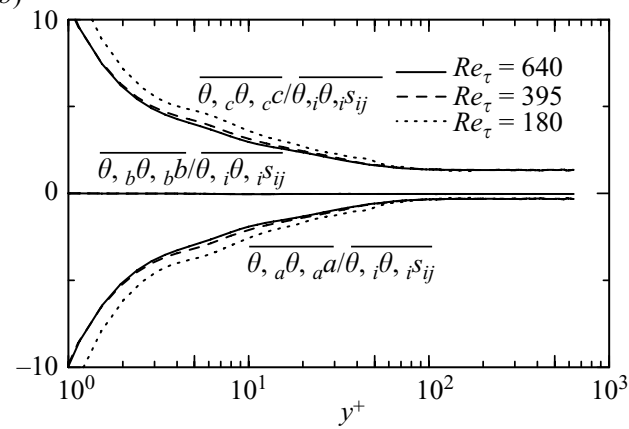

FIGURE 5. Turbulent production terms of $\overline{\omega_{i} \omega_{i}}$ and $\overline{\theta,{ }_{i} \theta,{ }_{i}}$ projected along each of the principal strain eigenvectors as functions of $y^{+}:$(a) $\overline{\omega_{\alpha} \omega_{\alpha} \alpha} / \overline{\omega_{i} \omega_{j} s_{i j}} \quad(\alpha \equiv a, b, c)$;

(b) $\overline{\theta,_{\alpha} \theta,_{\alpha} \alpha} / \overline{\theta,{ }_{i} \theta,{ }_{j} s_{i j}}(\alpha \equiv a, b, c)$.

Unlike the mean square values, the turbulent production terms projected along the principal strain rate directions are not simply inferred from figure 4 . The terms are normalized as follows:

$$
\overline{\omega_{\alpha} \omega_{\alpha} \alpha} / \overline{\omega_{i} \omega_{j} s_{i j}} \quad \text { or } \quad \overline{\theta,{ }_{\alpha} \theta,{ }_{\alpha} \alpha} / \overline{\theta,{ }_{i} \theta,{ }_{j} s_{i j}}(\alpha \equiv a, b, c),
$$

where $\left(\overline{\omega_{a} \omega_{a} a}+\overline{\omega_{b} \omega_{b} b}+\overline{\omega_{c} \omega_{c} c}\right) / \overline{\omega_{i} \omega_{j} s_{i j}} \equiv 1$ and $\left.\overline{\theta_{, a} \theta,_{a} a}+\overline{\theta_{,_{b}} \theta,_{b} b}+\overline{\theta, c} \theta,,_{c} c\right) /$ $\overline{\theta,{ }_{i} \theta,{ }_{j} s_{i j}} \equiv 1$. Their distributions are shown in figure 5. Near the wall, $2 \overline{\omega_{i} \omega_{j} s_{i j}}$ consists mostly of the component along the $b$ strain rate direction, whilst $-2 \overline{\theta,{ }_{i} \theta,{ }_{j} s_{i j}}$ contains mainly components along the $a$ and $c$ strain rate directions. This implies that the turbulent production terms also prefer to align with the mean strain rate. We should note that unlike $2 \overline{\omega_{i} \omega_{j} s_{i j}}$, the mean strain rate is not necessarily associated with the increase in $-2 \overline{\theta,{ }_{i} \theta,{ }_{j} s_{i j}}$, since the latter is only amplified by the $c$ strain rate. In the outer region, on the other hand, the relative contributions are almost the same as for isotropic turbulence (Vincent \& Meneguzzi 1994 for $2 \overline{\omega_{i} \omega_{j} s_{i j}}$; Vedula et al. 2001 for $\left.-2 \overline{\theta,{ }_{i} \theta,{ }_{j} s_{i j}}\right)$. In particular, the magnitudes of $\overline{\theta,{ }_{\alpha} \theta,{ }_{\alpha} \alpha} / \overline{\theta,{ }_{i} \theta,{ }_{j} s_{i j}}(\alpha \equiv a, b, c)$ are nearly identical with those of Vedula et al. (2001). Figure 5 also shows larger magnitudes near the wall than in the outer region especially for $\overline{\theta,{ }_{c} \theta,{ }_{c} c} / \overline{\theta,{ }_{i} \theta,{ }_{j} s_{i j}}$, suggesting that near the wall $\theta,{ }_{i}$ is more likely to be correlated with the $c$ strain rate than in isotropic turbulence. For isotropic turbulence, the strain rate and vorticity exhibit strong spatial and temporal intermittency (e.g. the Lagrangian study of Yeung 2001). These quantities thus become decorrelated in time quite rapidly. In particular, Yeung (2001) reported that the scalar dissipation rate becomes decorrelated more rapidly than the energy dissipation rate so that $\theta,{ }_{i}$ may not have enough time to align itself with the direction of the most compressive strain. This in turn can lead to weak correlations between the strain rate and $-2 \overline{\theta,{ }_{i} \theta,{ }_{j} s_{i j}}$. The scenario differs in the near-wall region due to the persistent space-filling nature of the near-wall organization (e.g. Johansson, Alfredsson \& Kim 1991) as well as the presence of the mean velocity and temperature gradients, leading to a stronger likelihood for $\theta,{ }_{i}$ to be correlated with the $c$ strain rate.

The effect of vorticity on the transport equation of $\overline{\theta,{ }_{i} \theta,,_{i}}$ (4.2) can be assessed in the same manner for isotropic turbulence (e.g. Gonzalez 2002; Brethouwer et al. 2003). For the present flow, the effect appears in the mean gradient production terms 


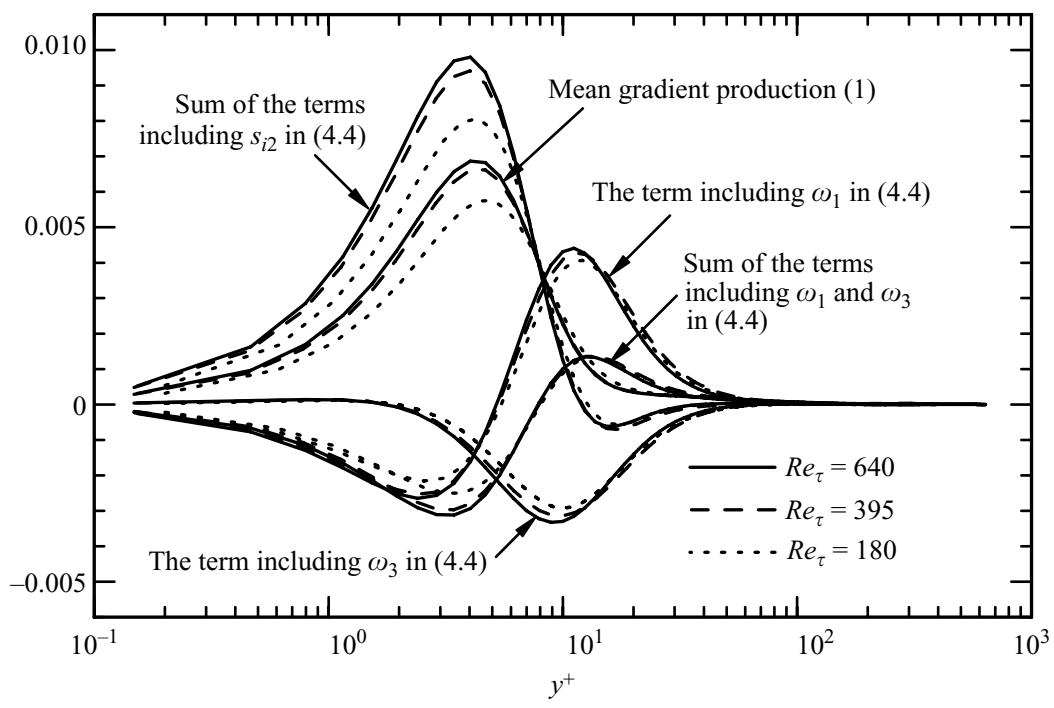

FIGURE 6. Effect of vorticity on the budget of $\overline{\theta,{ }_{i} \theta,{ }_{i}}((4.4)-$ the normalization is by wall units).

(1) and (2) (see (4.2)):

$$
\begin{aligned}
-2 \theta,,_{i}^{+} \frac{\partial u_{j}^{+}}{\partial x_{i}^{+}} \frac{\partial \bar{\Theta}^{+}}{\partial x_{j}^{+}}= & -2 \overline{\theta,{ }_{i}^{+} s_{i 2}^{+}} \frac{\partial \bar{\Theta}^{+}}{\partial x_{2}^{+}}+\left(-\overline{\theta,{ }_{1}^{+} \omega_{3}^{+}}+\overline{\theta,{ }_{3}^{+} \omega_{1}^{+}}\right) \frac{\partial \bar{\Theta}^{+}}{\partial x_{2}^{+}}, \\
& -2 \overline{\theta,_{i}^{+} \theta,,_{j}^{+}} \frac{\partial \bar{U}_{j}^{+}}{\partial x_{i}^{+}}=-2 \overline{\theta,,_{2}^{+} \theta,{ }_{1}^{+}}\left(\bar{S}_{12}^{+}+\bar{W}_{12}^{+}\right),
\end{aligned}
$$

where $S_{i j}\left(\equiv(1 / 2)\left(U_{i, j}+U_{j, i}\right)\right), W_{i j}\left(\equiv(1 / 2)\left(U_{i, j}-U_{j, i}\right)\right)$ are the instantaneous strain rate and rate of rotation tensors, respectively. As indicated in figure $3(b)$, the magnitudes of (4.4) and (4.5) are significant near the wall. Figure 6 illustrates that in (4.4), the term containing the strain rate is important in the viscous sublayer, whereas that with $\omega_{1}$ is important in the buffer region. The former is attributed to the significant increase in $\overline{\theta, 2} \theta, 2$ (see figure $1 b$ ), whilst the latter amplifies the magnitude of $\overline{\theta,{ }_{3} \theta, 3}$ and implies that the quasi-streamwise vortices are essential for enhancing the small-scale scalar mixing. In (4.4), the term containing $\omega_{3}$ is negative. Since $\omega_{3} \simeq-2 s_{12}=-2 s_{21}$, it is difficult to distinguish between the effects of the strain rate and those of the vorticity. The same difficulty is encountered with (4.5), since $\bar{S}_{12}=\bar{W}_{12}$ in this flow. The present results underline that, unlike isotropic turbulence in which the magnitudes of the mean gradient production terms are negligible (e.g. Ruetsch \& Maxey 1992; Vedula et al. 2001; Brethouwer et al. 2003), the effect of vorticity cannot be neglected near the wall. In the outer region, however, (4.4) can be neglected due to the small magnitude of the mean temperature gradient so that $\omega_{i} \omega_{i}$ and $\theta,{ }_{i} \theta,{ }_{i}$ are produced by the same type of straining motion, i.e. $2 \overline{\omega_{i} \omega_{j} s_{i j}}(4.1)$ and $-2 \overline{\theta,{ }_{i} \theta,{ }_{j} s_{i j}}$ (4.2), as for isotropic turbulence.

\section{Correlation coefficients between $\omega_{i}$ and $\theta,{ }_{i}$}

The similarity between $\overline{\omega_{i} \omega_{i}}$ and $\overline{\theta,{ }_{i} \theta,_{i}}(\S 3)$ and between the transport equations for these two quantities $(\S 4)$ suggests a strong correlation between $\omega_{i}$ and $\theta,{ }_{i}$. In this section, we attempt to quantify this via correlation coefficients. The relation 
(a)

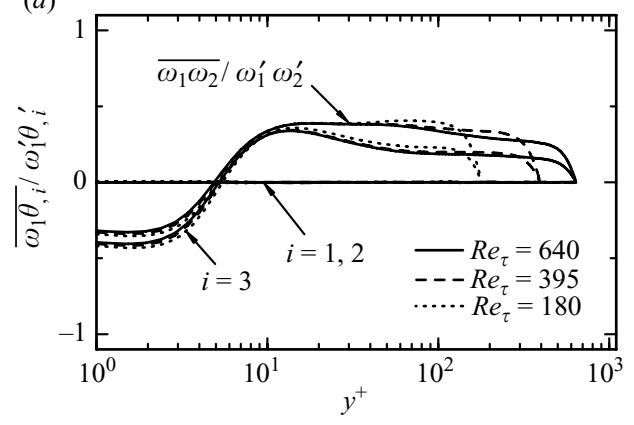

(b)

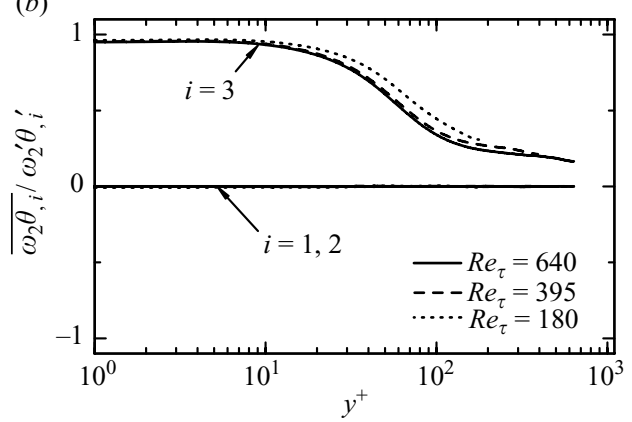

(c)

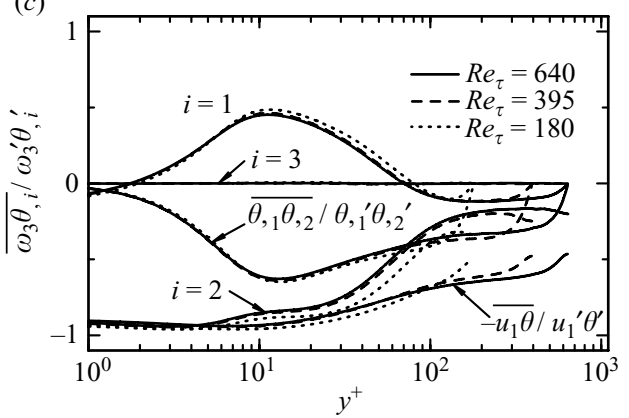

FIGURE 7. Correlation coefficients between vorticity components and temperature derivatives: (a) $\overline{\omega_{1} \theta, i} / \omega_{1}^{\prime} \theta,_{i}^{\prime} ;(b) \overline{\omega_{2} \theta,_{i}} / \omega_{2}^{\prime} \theta,_{i}^{\prime} ;$ (c) $\overline{\omega_{3} \theta,,_{i}} / \omega_{3}^{\prime} \theta,_{i}^{\prime}$. In $(a), \overline{\omega_{1} \omega_{2}} / \omega_{1}^{\prime} \omega_{2}^{\prime}$ obtained from the present study is included for comparison. In $(c), \overline{\theta,{ }_{1} \theta, 2} / \theta,{ }_{1}^{\prime} \theta,{ }_{2}^{\prime}$ and $\overline{u_{1} \theta} / u_{1}^{\prime} \theta^{\prime}$ obtained from the present study are included together with $\overline{u_{1} \theta} / u_{1}^{\prime} \theta^{\prime}$ by Kasagi et al. (1992) for $R e_{\tau}=150$.

between the correlations and the organized structures is also assessed by examining the instantaneous fields.

Figure 7 shows the correlation coefficients of $\overline{\omega_{i} \theta, j}$ together with those of $\overline{u_{1} \theta}$. Also included are the non-zero correlation coefficients of the vorticity and scalar gradient vectors, i.e. $\overline{\omega_{1} \omega_{2}}$ and $\overline{\theta,,_{1} \theta, 2}$. Of the nine correlations, only four $\left(\overline{\omega_{1} \theta, 3}, \overline{\omega_{2} \theta, 3}, \overline{\omega_{3} \theta, 1}, \overline{\omega_{3} \theta, 2}\right)$ are non-zero, whereas the other five are almost zero due to symmetry with respect to the $x_{3}$ direction. (At the centreline, the magnitudes of $\overline{\omega_{1} \theta, 3}$ and $\overline{\omega_{3} \theta, 1}$ are also almost zero due to symmetry with respect to both $x_{2}$ and $x_{3}$.) The magnitudes of the non-zero correlation coefficients, plotted against $y^{+}$, are quite large in the near-wall region. In particular, $\overline{\omega_{3} \theta, 2} / \omega_{3}^{\prime} \theta,_{2}^{\prime}$ is almost identical with $-\overline{u_{1} \theta} / u_{1}^{\prime} \theta^{\prime}$ very close to the wall, and the distribution of $\overline{\omega_{2} \theta, 3} / \omega_{2}^{\prime} \theta,_{3}^{\prime}$ follows that of $\overline{u_{1} \theta} / u_{1}^{\prime} \theta^{\prime}$ closely. Away from the wall, the magnitudes of the four non-zero correlation coefficients tend to decrease with increasing distance from the wall, and their magnitudes are close to each other. These results indicate that $\omega_{i}$ and $\theta,{ }_{i}$ are interlinked throughout the channel, although the level of correlation varies in the $x_{2}$ direction. Further, the dot product between the two vectors, i.e. the sum of $\overline{\omega_{1} \theta, 1}, \overline{\omega_{2} \theta, 2}$ and $\overline{\omega_{3} \theta, 3}$, is negligible everywhere in the channel (which is not shown here). This behaviour is similar to what has been observed in isotropic turbulence (Pumir 1994; Brethouwer et al. 2003). Whilst the orientations of $\omega_{i}$ and $\theta,{ }_{i}$ vary with increasing distance from the wall $(\S 4)$, the vorticity vector is perpendicular to the scalar gradient vector throughout the channel. However, this does not imply that the effect of the vorticity on small-scale mixing is negligible for the present flow. In fact, 

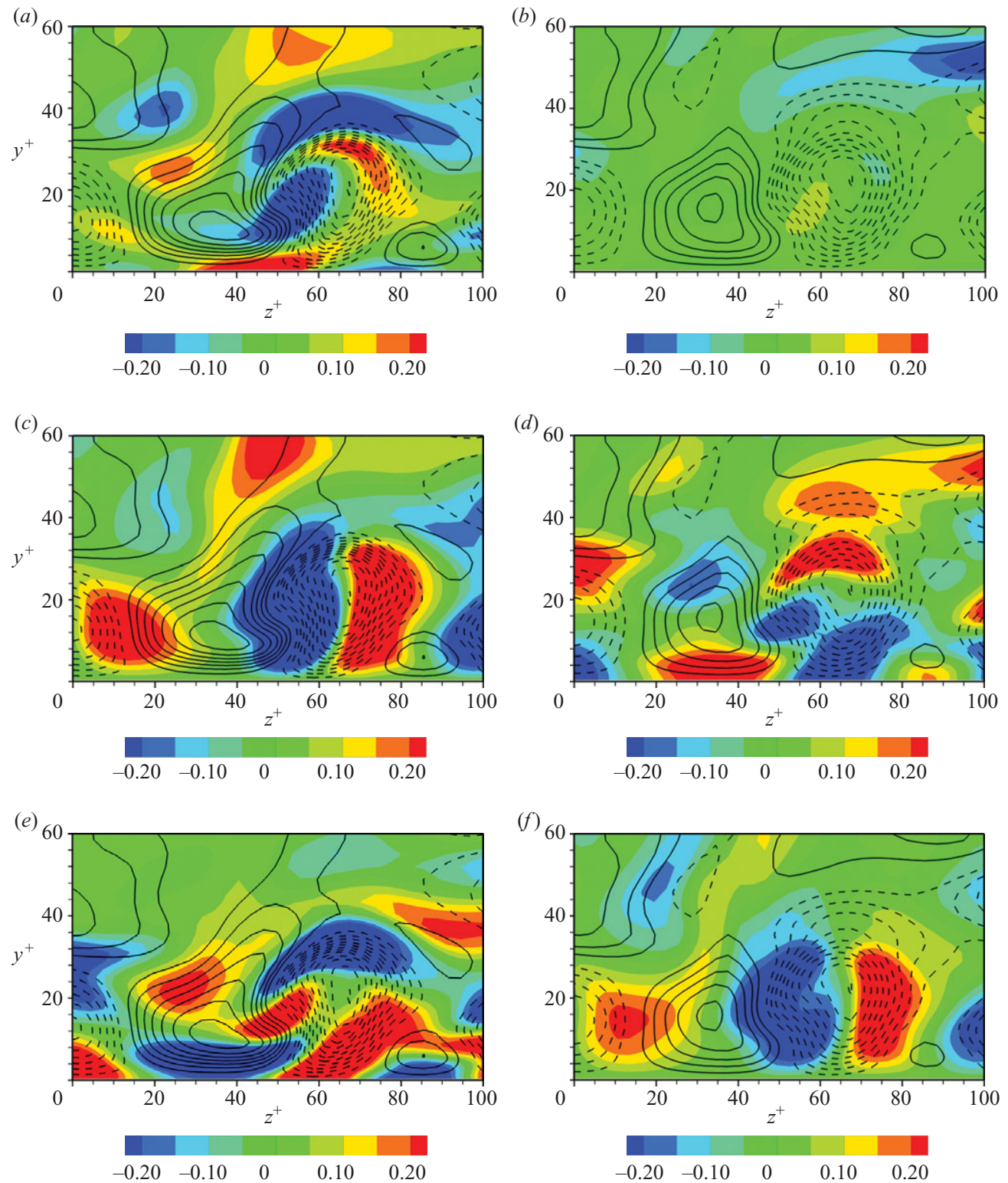

FIGURE 8. Instantaneous isocontours in the $y-z$ plane of vorticity and temperature derivative components for $R e_{\tau}=180$. The normalization is by wall units: (a) $\omega_{1}^{+} ;(b) \quad \theta,{ }_{1}^{+} ;(c) \omega_{2}^{+}$; (d) $\quad \theta,,_{2}^{+} ;(e) \quad \omega_{3}^{+} ;(f) \quad \theta,{ }_{3}^{+}$. Colour isocontours are used for $\omega_{i}$ and $\theta,{ }_{i}$, while lines are used for $u_{1}^{+}((a),(c),(e))$ and $\theta^{+}((b),(d),(f))$. (Solid and dashed lines refer to positive and negative values; the line increment is 0.5 .)

$\overline{\omega_{1} \theta, 3}$ is important near the wall, as discussed in $\S 4$ (see also (4.4)), but small in the outer region due to the negligibly small magnitude of the mean temperature gradient.

The implication of figure 7 is discussed in the context of turbulence structures. In the near-wall region, the large magnitude of $\overline{\omega_{3} \theta,_{2}} / \omega_{3}^{\prime} \theta,_{2}^{\prime}$ is consistent with the nearly perfect similarity between the momentum and thermal streaks (figure 8 ). There is also a strong correlation between $\overline{\omega_{2} \theta,_{3}} / \omega_{2}^{\prime} \theta,_{3}^{\prime}$ and the streaks, since concentrations 

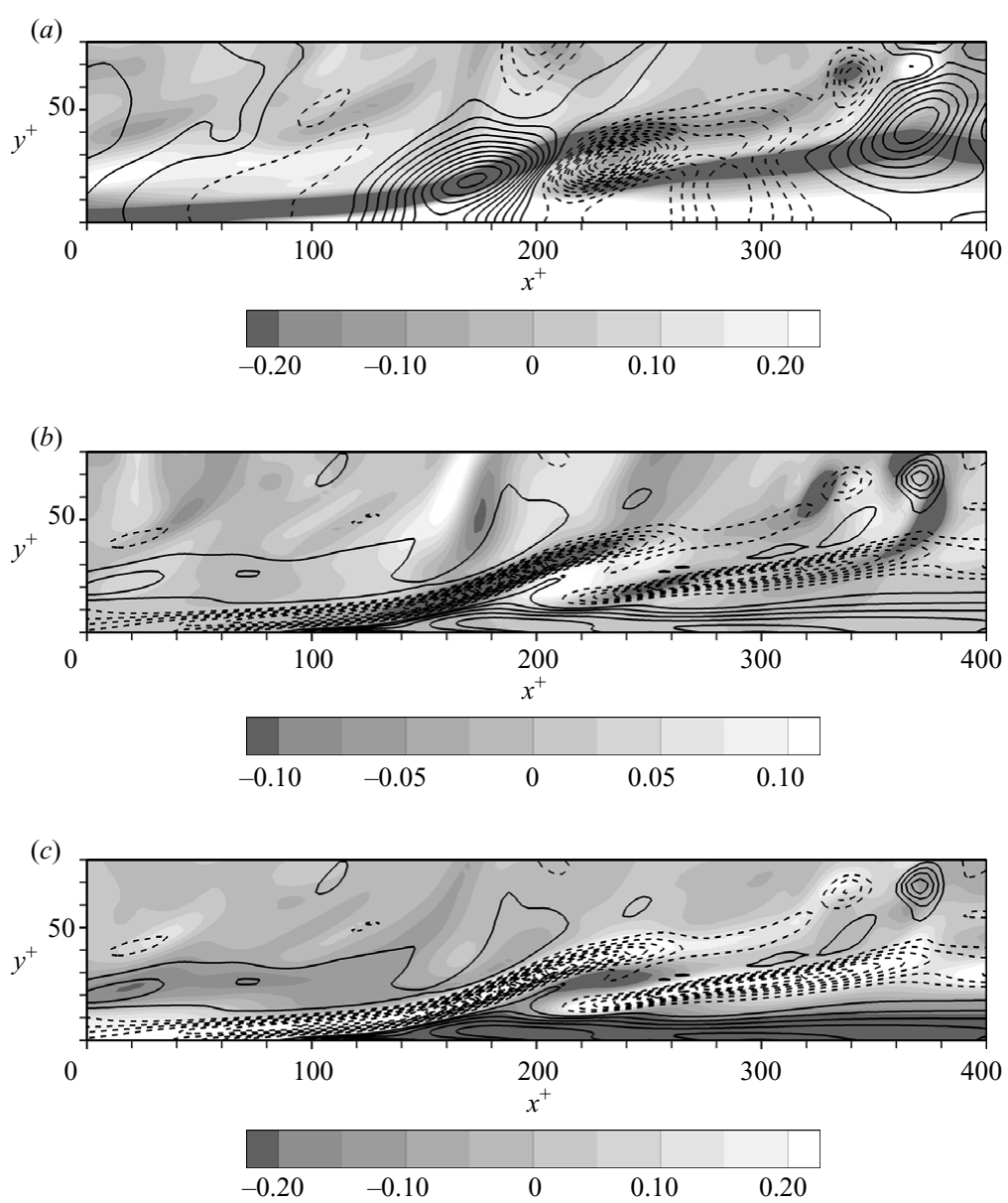

FIGURE 9. Instantaneous isocontours in the $x-y$ plane of vorticity and temperature derivative components for $R e_{\tau}=180$. The normalization is by wall units: (a) $\omega_{3}^{+} ;(b) \quad \theta,,_{1}^{+} ;(c) \quad \theta_{, 2}^{+}$. Isocontours are used for $\omega_{3}^{+}, \theta,{ }_{1}^{+}$and $\theta,,_{2}^{+}$. Lines are used for $p^{+}$in $(a)$ and $\omega_{3}^{+}$in $(b)$ and (c). (Solid and dashed lines refer to positive and negative values; the line increment is 0.5 in $(a)$ and 0.1 in $(b)$ and $(c)$.

of $\omega_{2}$ and $\theta,,_{3}$ occur at the interfaces between positive and negative $u_{1}$ or $\theta$ streaks. However, the streaks are not the only structures which contribute to $\overline{\omega_{1} \theta,,_{3}} / \omega_{1}^{\prime} \theta,_{3}^{\prime}$ and $\overline{\omega_{3} \theta, 1} / \omega_{3}^{\prime} \theta,_{1}^{\prime}$. The quasi-streamwise vortices (e.g. Robinson 1991) and internal shear layers (e.g. Jiménez et al. 1988; Johansson et al. 1991) are also likely contributors. The evidence appears in the instantaneous isocontours of $\omega_{i}$ and $\theta,_{i}$ shown in figures 8 and 9. In figure 8 , a counterclockwise vortex $\left(\omega_{1}<0\right)$ appears at $y^{+} \approx 20$, which is located at the interface between the positive and negative $u_{1}$ or $\theta$ streaks $\left(\omega_{2}<0\right.$ or $\theta, 3<0)$. This is consistent with $\overline{\omega_{1} \theta, 3} / \omega_{1}^{\prime} \theta,_{3}^{\prime}>0$. The opposite is true for the clockwise vortex $\left(\omega_{1}>0\right.$ ), where $\omega_{2}>0$ or $\theta,,_{3}>0$, and hence $\overline{\omega_{1} \theta,_{3}} / \omega_{1}^{\prime} \theta,_{3}^{\prime}>0$ (not shown here). The $\omega_{1}$ isocontours change sign close to the wall (figure $8 a$ ) due to the motion induced by the non-slip boundary condition at the wall (e.g. Kim, Moin \& Moser 1987; Robinson 1991). This results in a change in sign of $\overline{\omega_{1} \theta,_{3}} / \omega_{1}^{\prime} \theta,_{3}^{\prime}$ and $\overline{\omega_{1} \omega_{2}} / \omega_{1}^{\prime} \omega_{2}^{\prime}$ at $y^{+}=5$ (figure $7 a$ ). For the momentum and thermal internal shear layers (figure 9), similar features are exhibited as previously indicated by Kim (1988) 
(a)

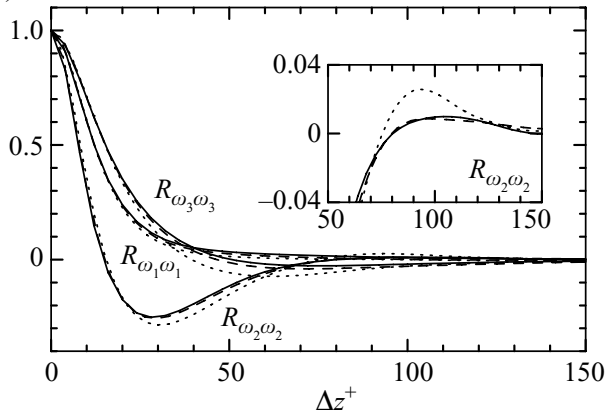

(b)

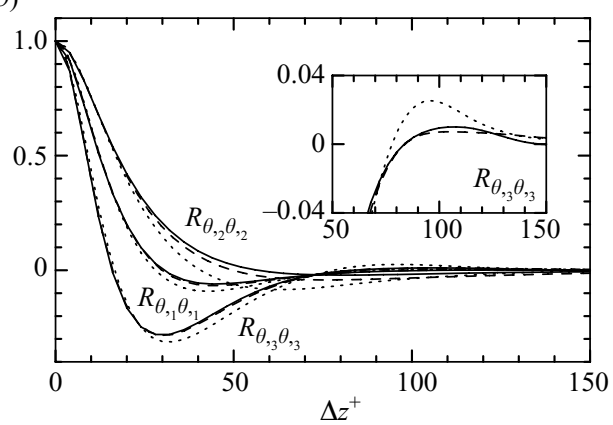

FIGURE 10. Two-point correlations of $\omega_{i}$ and $\theta,{ }_{i}$ in the $z$ direction for $R e_{\tau}=180,395$ and 640 : (a) $\quad R_{\omega_{i} \omega_{i}}(\Delta z) ;(b) \quad R_{\theta, i} \theta_{, i}(\Delta z)$. In each case, the fixed point is at $y^{+}=10$. In the insets, an exaggerated vertical scale is used to highlight the local positive maximum near $\Delta z^{+}=100$.

and Kim \& Moin (1989). The positive $u_{1}$ (or $\theta$ ) structure impacts the negative $u_{1}$ (or $\theta$ ) structure in the buffer region, and the pressure fluctuation $(p)$ is large and positive there. Consequently, regions with $\omega_{3}<0, \theta,,_{1}<0$ and $\theta,,_{2}>0$ appear near the wall, consistent with $\overline{\omega_{3} \theta,,_{1}} / \omega_{3}^{\prime} \theta,_{1}^{\prime}>0, \overline{\omega_{3} \theta,,_{2}} / \omega_{3}^{\prime} \theta,{ }_{2}<0$ and $\overline{\theta, 1} \theta,,_{2} / \theta,{ }_{1}^{\prime} \theta,{ }_{2}^{\prime}<0$. These results indicate that the large magnitudes of the correlation coefficients are closely associated with the near-wall organization. They also imply that the presence of the thermal streaks and internal shear layers are essential for mixing the scalar, whereas, in the light of (4.4), the quasi-streamwise vortices are important for enhancing the mixing as well as the strain rate.

One may expect that the near-wall behaviour of $\omega_{i}$ and $\theta,,_{i}$ does not change appreciably with increasing Reynolds number because the correlation coefficients are plotted against $y^{+}$. However, inspection of the instantaneous fields (not shown here) revealed that, near the wall, $\omega_{i}$ and $\theta,{ }_{i}$ change in the $x$ and $z$ directions with increasing $R e_{\tau}$. This may be associated with the fact that the near-wall momentum and thermal streaks become dense and clustered with increasing Reynolds numbers (see, for example, Robinson 1991; Abe et al. 2001, 2004a, b; Jiménez, del Álamo \& Flores 2004; Tanahashi et al. 2004; Iwamoto, Kasagi \& Suzuki 2005). To quantify this, two-point correlation coefficients of $\omega_{i}$ and $\theta,{ }_{i}$ defined as

$$
\begin{aligned}
R_{\omega_{i} \omega_{i}}\left(\Delta x_{i}\right) & =\overline{\omega_{i}\left(\Delta x_{i}\right) \omega_{i}\left(x_{i}+\Delta x_{i}\right)} / \omega_{i}\left(\Delta x_{i}\right)^{\prime} \omega_{i}\left(x_{i}+\Delta x_{i}\right)^{\prime}, \\
R_{\theta, i} \theta_{i}\left(\Delta x_{i}\right) & =\overline{\theta,{ }_{i}\left(\Delta x_{i}\right) \theta,{ }_{i}\left(x_{i}+\Delta x_{i}\right)} / \theta,{ }_{i}\left(\Delta x_{i}\right)^{\prime} \theta,,_{i}\left(x_{i}+\Delta x_{i}\right)^{\prime},
\end{aligned}
$$

are examined at $y^{+}=10$. Although only the spanwise two-point correlations are shown in figure 10, correlations in both $x$ and $z$ directions (e.g. $R_{\omega_{2} \omega_{2}}\left(\Delta x_{1}\right), R_{\theta, 3} \theta_{3}\left(\Delta x_{1}\right)$, $R_{\omega_{3} \omega_{3}}\left(\Delta x_{3}\right)$ and $\left.R_{\theta_{2,2} \theta_{2}}\left(\Delta x_{3}\right)\right)$ vary with increasing $R e_{\tau}$. In particular, the negative maximum of $R_{\omega_{3} \omega_{3}}\left(\Delta x_{3}\right)$ and $R_{\theta, 2 \theta, 2}\left(\Delta x_{3}\right)$ at $\Delta z^{+} \approx 50$ becomes less pronounced; the same is also true for $R_{u u}\left(\Delta x_{3}\right)$ and $R_{\theta \theta}\left(\Delta x_{3}\right)$ (not shown here). This is consistent with the present observation that, unlike those at $R e_{\tau}=180$, the $\omega_{3}$ and $\theta, 2$ isocontours at $R e_{\tau}=640$ (not shown here) exhibit dense and clustered structures and largescale patterns in the $z$ direction. At the wall, the same trend has been reported by Österlund (1999) for $R_{u u}\left(\Delta x_{3}\right)$ in a turbulent boundary layer and Abe et al. (2004b) for $R_{\theta \theta}\left(\Delta x_{3}\right)$ in a turbulent channel flow. In these papers, this behaviour was attributed to the effect of the large outer layer structures on the near-wall region. Interestingly, unlike $R_{\omega_{3} \omega_{3}}\left(\Delta x_{3}\right)$ and $R_{\theta_{2} \theta_{, 2}}\left(\Delta x_{3}\right), \quad R_{\omega_{2} \omega_{2}}\left(\Delta x_{3}\right)$ and $R_{\theta, 3,3}\left(\Delta x_{3}\right)$ show negative and positive peaks at $\Delta z^{+} \approx 30$ and $\Delta z^{+} \approx 110$, respectively (see figure 10 
and the insets). These peaks may be associated with the width of the streaks, since concentrations of $\omega_{2}$ and $\theta,{ }_{3}$ often appear at the spanwise interfaces between streaks (figure $8 c, f$ ). Robinson (1991), who examined the turbulent boundary layer DNS database of Spalart (1988), suggested that the width of the low-speed streaks ranges between 20 and 80 wall units, whereas that of the high-speed streaks is between 40 and 100 wall units. Since the low-speed streaks are dominant in the near-wall region, the negative peak at $\Delta z^{+} \approx 30$ may be related to the width of these streaks. The positive peak at $\Delta z^{+} \approx 110$ may be regarded as the width of both high- and low-speed streaks which appear alternately in the $z$ direction. Given that the magnitudes of the negative maxima of $R_{\omega_{2} \omega_{2}}\left(\Delta x_{3}\right)$ and $R_{\theta, 3 \theta, 3}\left(\Delta x_{3}\right)$ are larger and less dependent on $\operatorname{Re}_{\tau}$ than those of $R_{u u}\left(\Delta x_{3}\right)$ and $R_{\theta \theta}\left(\Delta x_{3}\right)$ (not shown here), $\omega_{2}$ and $\theta,_{3}$ may provide more accurate data for the spanwise dimension and separation between streaks than $u_{1}$ and $\theta$.

Away from the wall, the sign of $\overline{\omega_{3} \theta, 1} / \omega_{3}^{\prime} \theta,{ }_{1}^{\prime}$ changes at $y^{+}=70$ (figure $7 c$ ), which may be related to the change in the principal orientation of $\omega_{3}$. For a turbulent channel flow, Moin, Adrian \& Kim (1987) indicated a clear transition from streamwiseoriented wall events to inclined shear stress events in the vicinity of the buffer layer. Blackburn et al. (1996) suggested that near the wall the most intense vorticity tends to be organized into tubular-shaped structures with $x-y$ alignments ranging between $0^{\circ}$ and $45^{\circ}$ to the wall, the alignment of $45^{\circ}$ being consistent with the direction of the mean strain rate (Moin \& Kim 1985), whereas in the outer region it lies mainly along the spanwise orientation. For a turbulent boundary layer, Robinson (1991) suggested that shear layers are prominent below $y^{+}=80$, whilst transverse vortices dominate above $y^{+}=80$.

In the outer layer, large-scale velocity and temperature structures appear frequently (e.g. Brown \& Thomas 1977; Chen \& Blackwelder 1978). Antonia \& Van Atta (1979) suggested that the anisotropic temperature fronts cause the non-zero skewness of the temperature derivatives in a turbulent boundary layer. Tanahashi et al. (2004) suggested a close association between the large-scale $u_{1}$ structures and $\omega_{1}$ in a turbulent channel flow. The present DNS data also throw some light on the relationship between small-scale $\left(\omega_{i}\right.$ and $\left.\theta_{, i}\right)$ and large-scale $\left(u_{1}\right.$ and $\left.\theta\right)$ structures. Figure 11 displays isocontours of $\omega_{3}$ and $\theta, 2$ in the $x-y$ plane together with those of $u_{1}$ and $\theta$. The $\omega_{3}$ and $\theta, 2$ concentrations occur at the interfaces of the large-scale $u_{1}$ and $\theta$ structures, suggesting that the large-scale $\theta$ structures are substantially responsible for the small-scale scalar mixing (see also $\S 6$ ). However, the similarity between the large-scale $u_{1}$ and $\theta$ structures is not as good as that between the near-wall $u_{1}$ and $\theta$ streaks, since the inclination to the wall of the $\theta$ contours is steeper than that of the $u_{1}$ contours in the outer region (see also the two-point correlations in the $x-y$ plane by Kawamura et al. 2002; Antonia et al. 2008). These results seem to be in accord with the fact that $\overline{\omega_{i} \theta,{ }_{j}} / \omega_{i}^{\prime} \theta,_{j}^{\prime}$ is smaller in the outer layer than in the near-wall region (see figure 7). From these considerations and the persistence of the structures (e.g. Johansson et al. 1991), one may surmise that $\omega_{i}$ and $\theta,_{i}$ are associated with the organized motions throughout the channel.

In figure 11 , in contrast to the $\omega_{3}$ contours, the $\theta, 2$ contours exhibit sheetlike structures in the outer region. Evidence for such structures has been found in several turbulent flows, e.g. homogeneous isotropic turbulence (e.g. Ruetsch \& Maxey 1992; Pumir 1994; Vedula et al. 2001; Brethouwer et al. 2003), homogeneous sheared turbulence (e.g. Nomura \& Elghobashi 1992) and turbulent shear flows (e.g. Prasad \& Sreenivasan 1990; Sreenivasan 1991; Buch \& Dahm 1998; Su \& Clemens 2003). They are aligned with the most compressive strain rate (Ashurst et al. 1987) and 

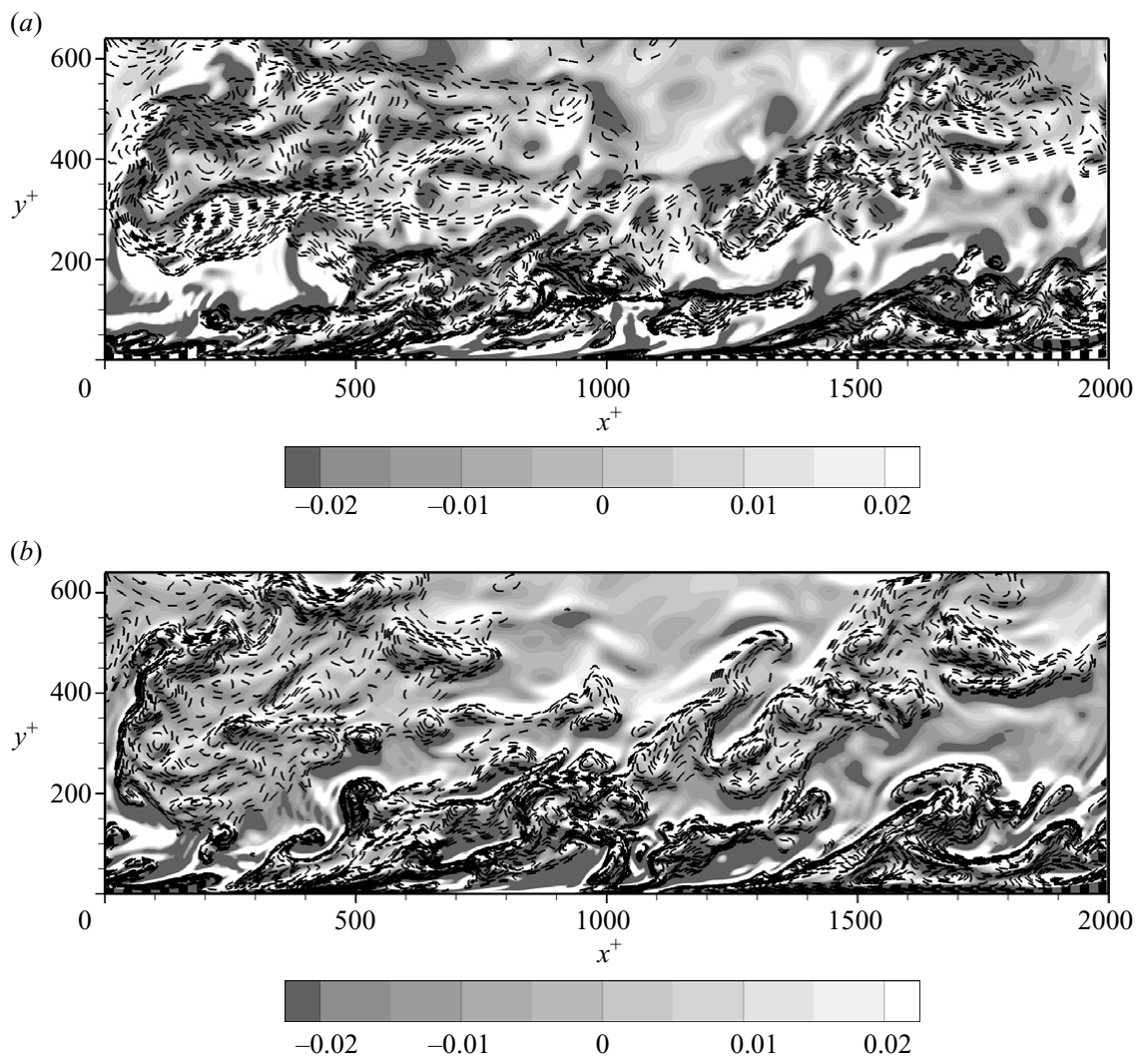

FIGURE 11. Instantaneous isocontours in the $x-y$ plane of the spanwise vorticity fluctuation and the wall-normal temperature derivative for $R e_{\tau}=640$. The normalization is by wall units: (a) $\omega_{3}^{+} ;(b) \quad \theta,{ }_{2}^{+}$. Isocontours are used for $\omega_{3}^{+}$and $\theta,,_{2}^{+}$, while lines are used for $u_{1}^{+}$and $\theta^{+}$. (Dashed lines refer to negative values; the line increment is 0.2.)

are associated with ramp-cliff structures (e.g. Sreenivasan, Antonia \& Britz 1979; Antonia et al. 1986; Holzer \& Siggia 1994). An estimate of their thickness $\lambda_{D x_{i}}$ can be inferred from the first zero-crossing location of the present two-point correlation of $\theta,{ }_{i}$ with a separation in the $x_{i}$ direction, $R_{\theta, i} \theta_{, i}\left(\lambda_{D x_{i}}\right)=0$, and is given in figure 12 . Note that this approach only provides a rough estimate for the thickness of the $\theta,{ }_{i}$ concentration in view of the three-dimensionality of the structures; in particular, the thickness of the $\theta,{ }_{1}$ concentration may be affected noticeably by the inclination of the structures. Near the wall, $\lambda_{D x_{i}}$ differs significantly between the two normalizations used in figure 12, reflecting the influence of the near-wall organization. Away from the wall, the three estimates of the thickness tend to collapse when Kolmogorov scaling is used. The success of this scaling is consistent with the observation that the thickness of the sheetlike structures also exhibits Kolmogorov scaling (Buch \& Dahm 1998; Moisy et al. 2001; Brethouwer et al. 2003; Su \& Clemens 2003). For $y^{+}>100$, the magnitudes are approximately constant, equal to about $6 \eta$ or $7 \eta_{B}$ (figure 12b). Ruetsch \& Maxey (1992) and Brethouwer et al. (2003) reported values of $4 \eta$ and 4 $\eta_{B}$, respectively, in isotropic turbulence, whilst Su \& Clemens (2003) reported a value of $5.4 \eta_{B}$ in a turbulent plane jet, the latter being closer to the present estimate. In a turbulent plane jet, there is evidence to suggest that the thickness scales on outer velocity and length scales (Buch \& Dahm 1998; Su \& Clemens 2003). This does not 

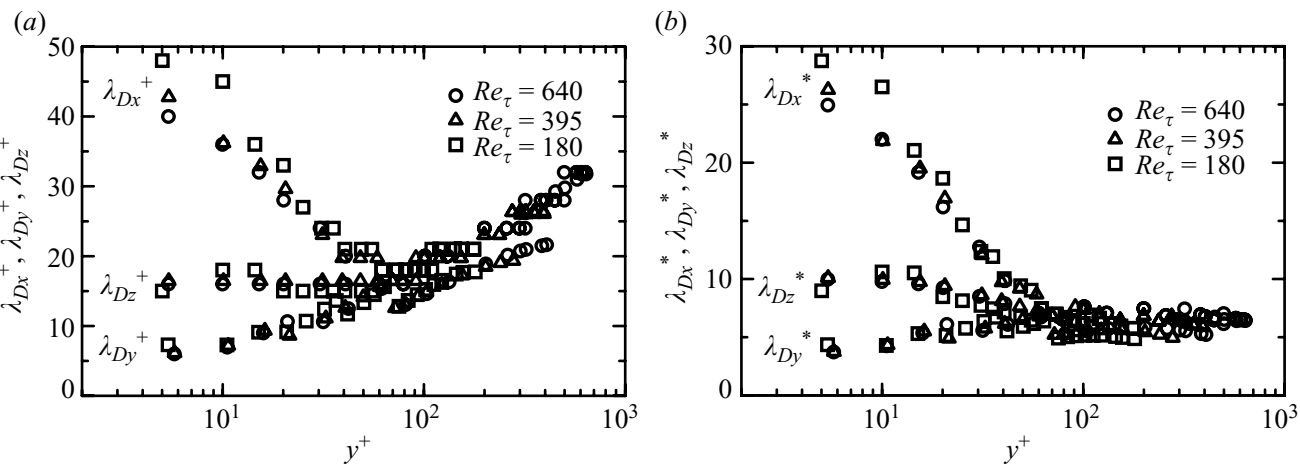

FiguRE 12. Distributions of the scalar dissipation thicknesses, $\lambda_{D x}, \lambda_{D y}, \lambda_{D z}$, estimated from the first zero-crossing point of $R_{\theta, 1} \theta_{, 1}\left(\Delta x_{1}\right), R_{\theta, 2 \theta, 2}\left(\Delta x_{2}\right)$ and $R_{\theta, 3 \theta, 3}\left(\Delta x_{3}\right)$, respectively, for $R e_{\tau}=180,395$ and 640 as functions of $y^{+}:(a)$ normalization by wall units; (b) normalization by Kolmogorov scales.

seem to be the case for a turbulent channel flow, since unlike a turbulent jet, the Kolmogorov scales vary significantly in the $x_{2}$ direction.

\section{Topology of the scalar dissipation rate}

The topology of small-scale structures has been examined intensively in isotropic turbulence. The most intense vorticity was found to reside in tubes, whereas moderate magnitudes of vorticity were associated with sheets (e.g. She et al. 1990; Ruetsch \& Maxey 1991; Jiménez et al. 1993; Vincent \& Meneguzzi 1994). On the other hand, intense scalar dissipation rates were sheetlike in form, as discussed in $\S 5$. Blackburn et al. (1996) suggested that in the outer region of a turbulent channel flow, the fine scale motion has many characteristics in common with various other turbulent flows. In this section, we investigate the topology of the scalar dissipation rate by examining its relationship with the enstrophy, energy dissipation rate and vortical structures, using instantaneous visualizations and conventional statistics.

Instantaneous isocontours of $\omega_{i} \omega_{i}, \varepsilon, Q\left(\equiv\left(\omega_{i} \omega_{i} / 4-s_{i j} s_{i j} / 2\right)=-u_{i, j} u_{j, i} / 2\right)$ and $\theta,{ }_{i} \theta,{ }_{i}$ are shown in the $y-z$ plane in figures 13 and 14 , where $Q$ denotes the second invariant of the velocity gradient tensor, and its positive value (with the vorticity dominant) corresponds to vortices (e.g. Robinson 1991). In a turbulent channel flow, organized structures such as streaks, internal shear layers and backs (or fronts) of the large-scale structures are intrinsic elements of the topology for the scalar dissipation rate. Close to the wall, there are strong concentrations of $\theta,{ }_{i} \theta,{ }_{i}$ parallel to the wall and elongated in the $x_{1}$ direction. (They are not shown here, since they are virtually coincident with the thermal streaks, a feature that has been well documented in the literature.) Slightly away from the wall, these concentrations tend to be annular in shape and seem to occur along the periphery of streaks (figure 13). They are space filling (figures 13 and 14) and aligned at about $45^{\circ}$ to the extensional (a) and compressive $(c)$ strain rate directions (not shown here; see $\S 3$ ). The similarity between $\omega_{i} \omega_{i}$ and $\theta,{ }_{i} \theta,,_{i}$ is excellent due to the near-wall similarity between $\omega_{i}$ and $\theta,{ }_{i}$ (see $\S 5$ ). The correlations among $\omega_{i} \omega_{i}, \varepsilon, Q, \theta,{ }_{i} \theta,{ }_{i}$ are also high, since $\omega_{i} \omega_{i} \approx \varepsilon$, and the quasi-streamwise vortices $(Q>0)$ are associated with high-energy dissipation rate sites (Robinson 1991). Further away from the wall, the internal shear layers and backs (or fronts) contribute significantly to $\theta,{ }_{i} \theta,,_{i}$ (figure 14). The strong $\theta,{ }_{i} \theta,,_{i}$ 

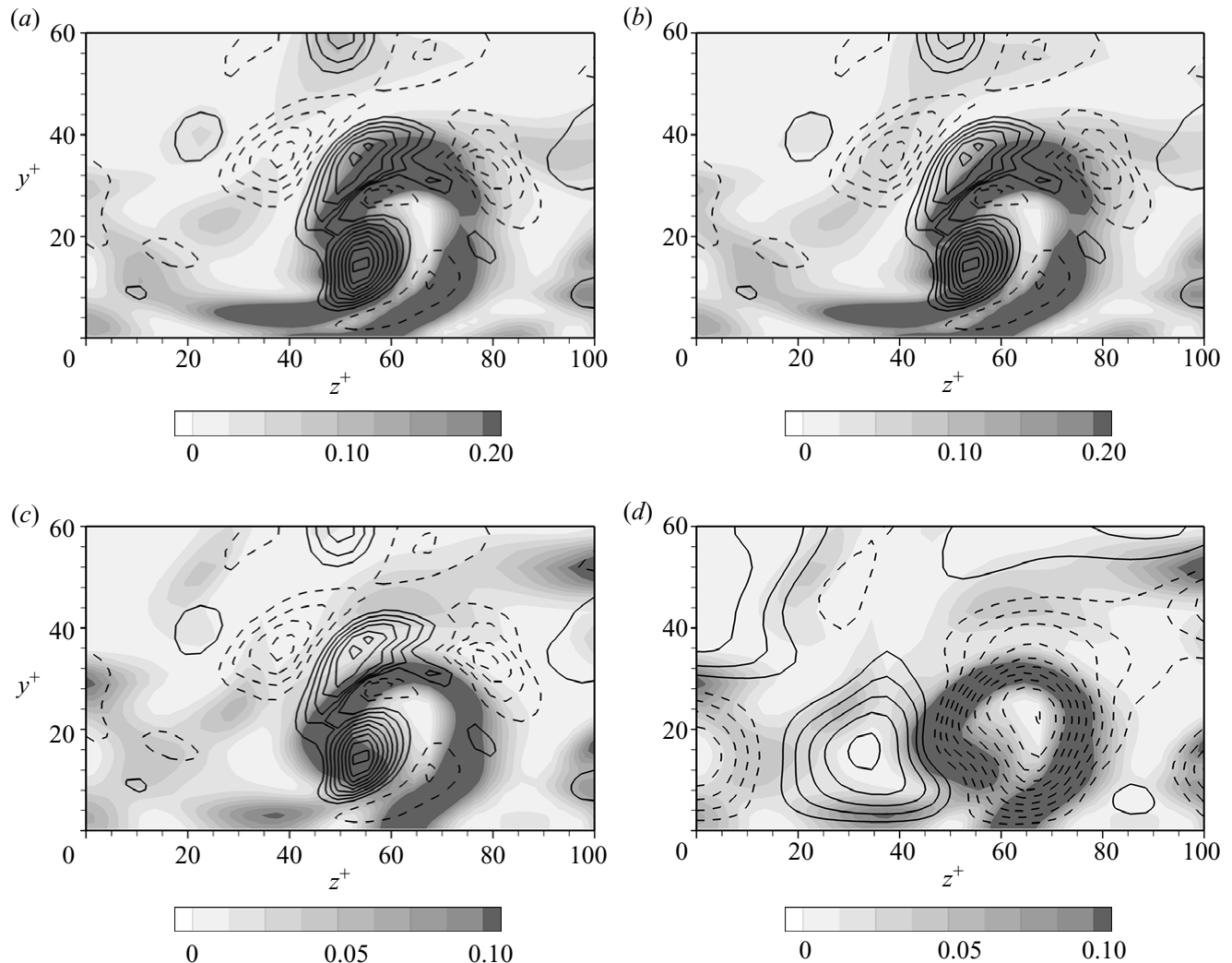

FIGURE 13. Instantaneous isocontours in the $y-z$ plane of the enstrophy, the energy dissipation rate, the scalar dissipation rate and the second invariant of the velocity gradient tensor for $R e_{\tau}=180$. The normalization is by wall units: (a) $\omega_{i}^{+} \omega_{i}^{+} ;(b) \varepsilon^{+} ;(c),(d) \quad \theta,{ }_{i}^{+} \theta,{ }_{i}^{+}$. Isocontours are used for $\omega_{i}^{+} \omega_{i}^{+}, \varepsilon^{+}$and $\theta,{ }_{i}^{+} \theta,{ }_{i}^{+}$, while lines are used for $Q^{+}((a)-(c))$ and $\theta^{+}(d)$. (Solid and dashed lines refer to positive and negative values; the line increment is 0.5 for $\theta^{+}$and 0.005 for $Q^{+}$.)

regions are spatially intermittent (spotty) structures (figure 14) and aligned with the most compressive $(c)$ strain rate (not shown here). In particular, they tend to appear at the edges of large-scale $\theta$ structures (figure $14 d$ ), which are sheetlike in form as discussed in $\S 5$ (see also figure 11). Blackburn et al. (1996) suggested a degree of similarity at small scales between the structures in isotropic turbulence and those in the outer region of a channel flow. This is also true for the scalar dissipation rate. For example, the structures for isotropic turbulence (e.g. Kerr 1985; Ruetsch \& Maxey 1991, 1992; Vedula et al. 2001) are observed in figure 14 (see, for example, $y^{+}=500$ and $z^{+}=700$ in this figure). The intense $\omega_{i} \omega_{i}$ regions correspond to $Q>0$ (figure 14a) and may be associated with the vortex tubes (e.g. She et al. 1990; Vincent \& Meneguzzi 1994). The intense $\varepsilon$ and $\theta,{ }_{i} \theta,{ }_{i}$ concentrations wrap around the positive $Q$ regions with an annular shape (figure $14 b, c$ ), but the overlap between $\varepsilon$ and $\theta,{ }_{i} \theta,{ }_{i}$ is small. It should be pointed out that, in the present flow, the intense $\theta,{ }_{i} \theta,{ }_{i}$ is not necessarily associated with the vorticity, energy dissipation rate and vortices but with the large-scale $\theta$ structures (see figures $11 b$ and $14 d$ ). This is consistent with the observation established in previous work (e.g. Sreenivasan et al. 1979, Antonia et al. 

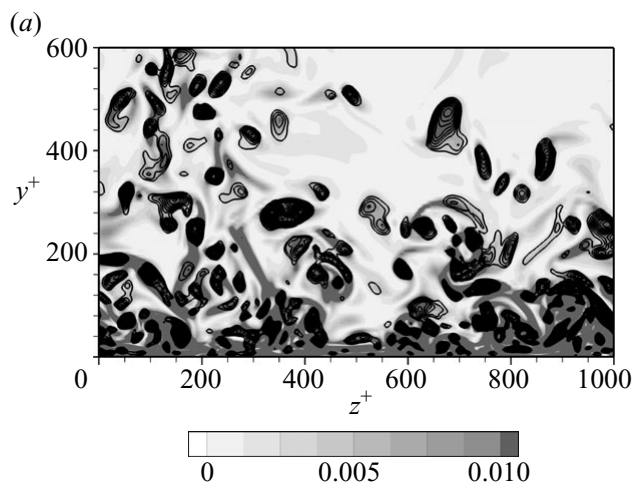

(c)

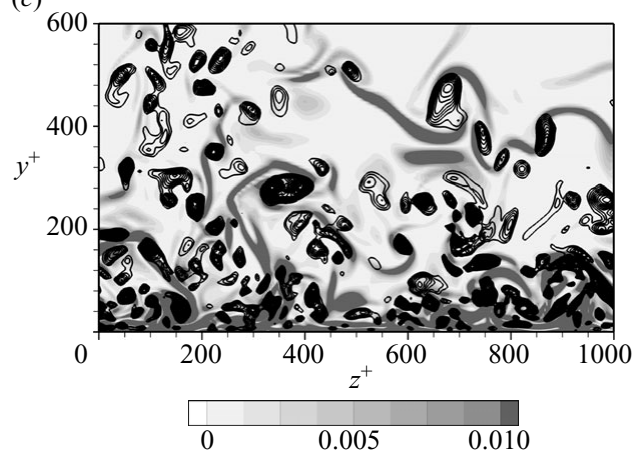

(b)

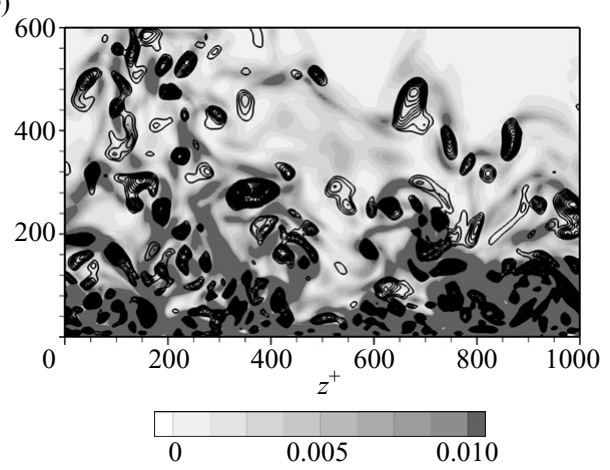

(d)

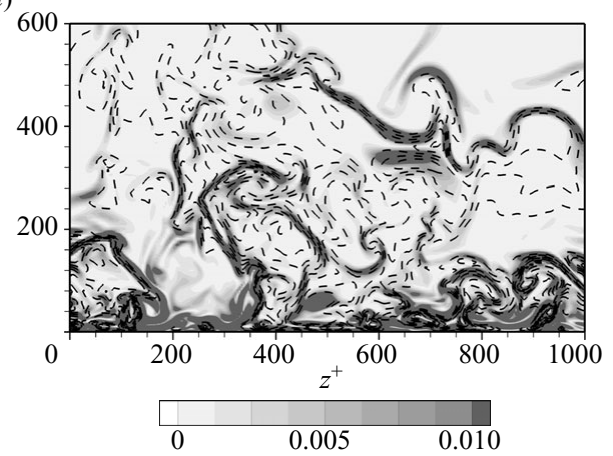

FIGURE 14. Instantaneous isocontours in the $y-z$ plane of the enstrophy, the energy dissipation rate, the scalar dissipation rate and the second invariant of the velocity gradient tensor for $R e_{\tau}=640$. The normalization is by wall units: (a) $\omega_{i}^{+} \omega_{i}^{+} ;(b) \varepsilon^{+} ;(c),(d) \quad \theta,{ }_{i}^{+} \theta,{ }_{i}^{+}$. Isocontours are used for $\omega_{i}^{+} \omega_{i}^{+}, \varepsilon^{+}$and $\theta,{ }_{i}^{+} \theta,{ }_{i}^{+}$, while lines are used for $Q^{+}$and $\theta^{+}$. In $(a)-(c)$, isocontours of the positive $Q^{+}$are shown, while in $(d)$, those of the negative $\theta^{+}$are shown. (Solid and dashed lines refer to positive and negative values; the line increment is 0.5 for $\theta^{+}$and 0.0005 for $Q^{+}$.)

1986; Sreenivasan 1990; Nomura \& Elghobashi 1992) that the most intense scalar dissipation rates are related to large-scale anisotropic structures of $\theta$.

A quantitative measure of the correlation between $\omega_{i} \omega_{i}$ and $\theta,{ }_{i} \theta,{ }_{i}$ is given in figure 15, where the non-centred correlation coefficient $\overline{\omega_{i}^{2} \theta,{ }_{j}^{2}} /\left(\overline{\omega_{i}^{2}} \cdot \overline{\theta,},_{j}^{2}\right)$ is shown. Also included are $\overline{\varepsilon \theta,{ }_{i}^{2}} /\left(\bar{\varepsilon} \cdot \overline{\theta,{ }_{i}^{2}}\right)$ and $\overline{\omega_{i}^{2} \varepsilon} /\left(\overline{\omega_{i}^{2}} \cdot \bar{\varepsilon}\right)$. Note that these correlations are related to the flatness factor of velocity-scalar mixed derivatives (Kerr 1985; Yeung, $\mathrm{Xu} \&$ Sreenivasan 2002). With this definition, $\overline{\omega_{i}^{2} \theta,{ }_{j}^{2}} /\left(\overline{\omega_{i}^{2}} \cdot \overline{\theta,}{ }_{j}^{2}\right)=1$ implies independence between $\omega_{i} \omega_{i}$ and $\theta,{ }_{i} \theta,{ }_{i}$ and therefore a zero correlation between $\omega_{i}$ and $\theta,{ }_{i}$ (Kerr 1985). The present value of $\overline{\omega_{i}^{2} \theta,_{j}^{2}} /\left(\overline{\omega_{i}^{2}} \cdot \overline{\theta,{ }_{j}^{2}}\right)$ is significantly greater than 1 across the channel, in contrast with the value of about 1 in isotropic turbulence (Kerr 1985). The Reynolds-number dependence is less likely to be observed between $R e_{\tau}=395$ and $R e_{\tau}=640$ than between $R e_{\tau}=180$ and $R e_{\tau}=395$, due to the low Reynolds-number effects (Antonia \& Kim 1994a). Close to the wall, the largest magnitude appears, and the similarity among $\overline{\omega_{i}^{2} \theta,{ }_{j}^{2}} /\left(\overline{\omega_{i}^{2}} \cdot \overline{\theta,{ }_{j}^{2}}\right), \overline{\varepsilon \theta,{ }_{i}^{2}} /\left(\bar{\varepsilon} \cdot \overline{\theta,{ }_{i}^{2}}\right)$ and $\overline{\omega_{i}^{2} \varepsilon} /\left(\overline{\omega_{i}^{2}} \cdot \bar{\varepsilon}\right)$ is excellent, consistent with the instantaneous visualizations in figure 13. Away from the wall, the magnitude decreases with increasing $y^{+}$, where the magnitude of $\overline{\varepsilon \theta,{ }_{i}^{2}} /\left(\bar{\varepsilon} \cdot \bar{\theta},{ }_{i}^{2}\right)$ is larger than that of $\overline{\omega_{i}^{2} \theta,{ }_{j}^{2}} /\left(\overline{\omega_{i}^{2}} \cdot \overline{\theta, 2}\right)$. This result suggests that $\theta,{ }_{i} \theta,{ }_{i}$ is better correlated with $\varepsilon$ (or with the strain rate) than with $\omega_{i} \omega_{i}$, which agrees with that in box turbulence with 

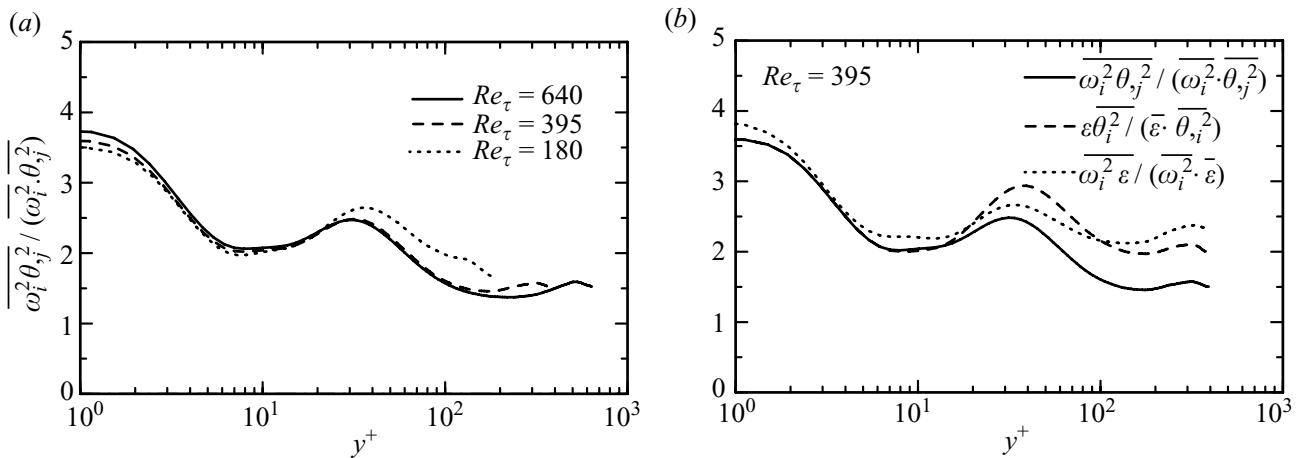

FIGURE 15. Distributions of correlations between $\omega_{i} \omega_{i}, \varepsilon, \theta,{ }_{i} \theta,{ }_{i}$ and $Q:(a) \quad \overline{\omega_{i}^{2} \theta,{ }_{j}^{2}} /\left(\overline{\omega_{i}^{2}} \cdot \overline{\theta,{ }_{j}^{2}}\right)$ for $R e_{\tau}=180,395$ and 640; (b) $\overline{\omega_{i}^{2} \theta,{ }_{j}^{2}} /\left(\overline{\omega_{i}^{2}} \cdot \overline{\theta,}{ }_{j}^{2}\right), \overline{\varepsilon \theta,{ }_{i}^{2}} /\left(\bar{\varepsilon} \cdot \overline{\theta,{ }_{i}^{2}}\right)$ and $\overline{\omega_{i}^{2} \varepsilon} /\left(\overline{\omega_{i}^{2}} \cdot \bar{\varepsilon}\right)$ for $R e_{\tau}=395$.

a constant mean scalar gradient (Kerr 1985; Ruetsch \& Maxey 1991, 1992; Pumir 1994; Vedula et al. 2001; Brethouwer et al. 2003). The local peak at $y^{+}=30$ does not correspond to the centroid of the quasi-streamwise vortices (Kim et al. 1987) but rather to the average height of the streaks (figures 8 and $13 d$ ). In this context, Smith \& Metzler (1983) suggested that the momentum streaks are barely noticeable for $y^{+}>40$. In the outer region, the magnitude is half that in the near-wall region. For a homogeneous shear flow, Nomura \& Elghobashi (1992) examined $\overline{\omega_{i}^{2} \theta,{ }_{j}^{2}} /\left(\overline{\omega_{i}^{2}} \cdot \overline{\theta,}{ }_{j}^{2}\right)$ and reported values of 1.55 and 1.58 when $S^{*}=0.15$ and 0.14 , respectively $\left(S \equiv\left(\mathrm{d} \bar{U}_{1} / \mathrm{d} x_{2}\right)\right)$. In the present flow, this magnitude of $S^{*}$ occurs at $y^{+} \approx 100$ (the distribution of $S^{*}$ is not shown here). At this location, the magnitude of $\overline{\omega_{i}^{2} \theta,{ }_{j}^{2}} /\left(\overline{\omega_{i}^{2}} \cdot \overline{\theta,{ }_{j}^{2}}\right)$ is nearly identical to that of Nomura \& Elghobashi (1992).

It is more common to consider (centred) correlation coefficients defined by

$$
\rho_{\phi \varphi} \equiv \frac{\overline{(\phi-\bar{\phi})(\varphi-\bar{\varphi})}}{\sigma_{\phi} \sigma_{\varphi}},
$$

where $\phi$ and $\phi$ denote either $\theta,{ }_{i} \theta,{ }_{i}, \omega_{i} \omega_{i}$ or $\varepsilon$ and $\sigma$ is the standard deviation of the centred variables. The correlation coefficients $\left(\rho_{\omega_{i}^{2} \theta,{ }_{j}}\right.$ and $\left.\rho_{\varepsilon \theta,{ }_{i}}\right)$ are non-zero throughout the channel (the distributions are not shown here). Their magnitudes are approximately the same (about 0.8) near the wall, whereas $\rho_{\varepsilon \theta,{ }_{i}^{2}}$ is larger than $\rho_{\omega_{i}^{2} \theta,{ }_{j}}$ away from the wall, the trend being consistent with that in the correlations (figure 15). At the centreline, $\rho_{\omega_{i}^{2} \theta_{, j}^{2}}$ and $\rho_{\varepsilon \theta,{ }_{i}^{2}}$ are about 0.1 and 0.3 , respectively. The magnitude of $\rho_{\varepsilon \theta, 2}$ was reported in several turbulent flows, e.g. turbulent jet (Antonia \& Van Atta 1975), atmospheric surface layer (Antonia \& Chambers 1980), grid turbulence (Zhou \& Antonia 2000) and isotropic turbulence (Wang, Chen \& Brasseur 1999; Antonia \& Orlandi 2003). The present value of $\rho_{\varepsilon \theta, 2}$ at the centerline is about twice as large as for isotropic turbulence (Wang et al. 1999; Antonia \& Orlandi 2003), suggesting that $\theta,{ }_{i} \theta,{ }_{i}$ is better correlated with $\varepsilon$ in a turbulent channel flow than in isotropic turbulence. This result is most likely associated with a larger degree of anisotropy in a turbulent channel flow than in isotropic turbulence as discussed in $\S 3$.

The correlations are further quantified by examining the joint p.d.f.s among $\omega_{i} \omega_{i}, \varepsilon, Q$ and $\theta,{ }_{i} \theta,{ }_{i}$ and are given in figure 16 , where the shapes of the distributions between $\omega_{i} \omega_{i}$ and $\varepsilon$ agree with those of Blackburn et al. (1996) (figure 16a,b). Note that these p.d.f.s are obtained from one instantaneous realization as in Blackburn et al. (1996). Near the wall, there are strong correlations among $\omega_{i} \omega_{i}, \varepsilon$, and $\theta,{ }_{i} \theta,{ }_{i}$, consistent with the correlations (figure 15), the correlation coefficients (not shown 
(a)

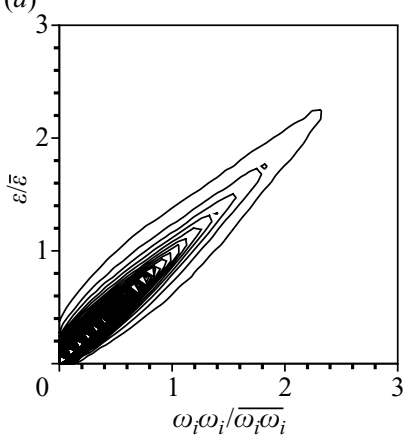

(d)

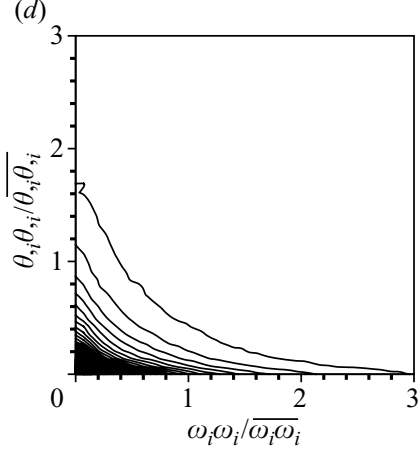

(b)

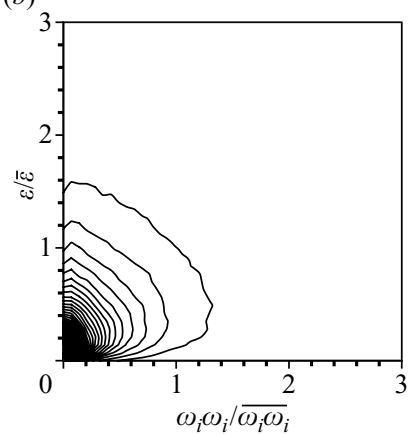

(e)

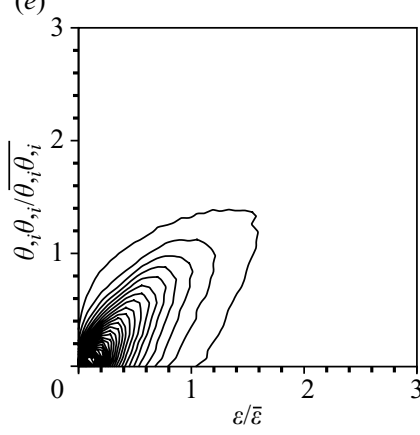

(c)

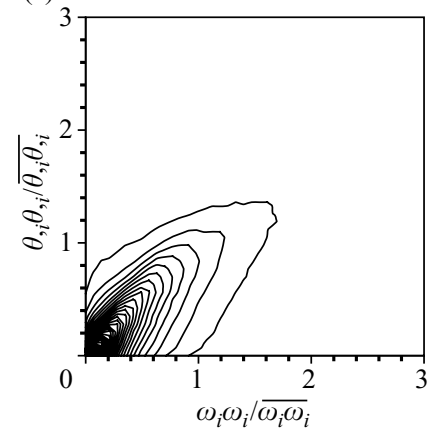

(f)

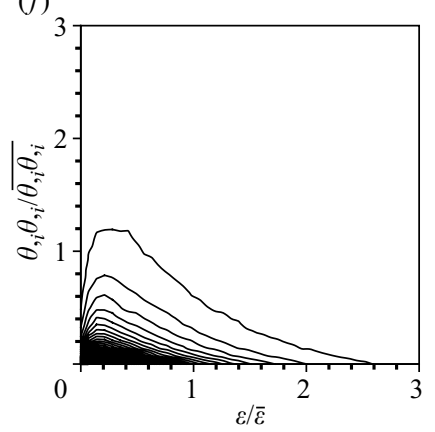

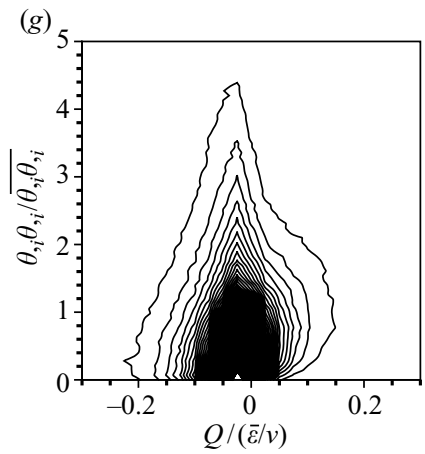

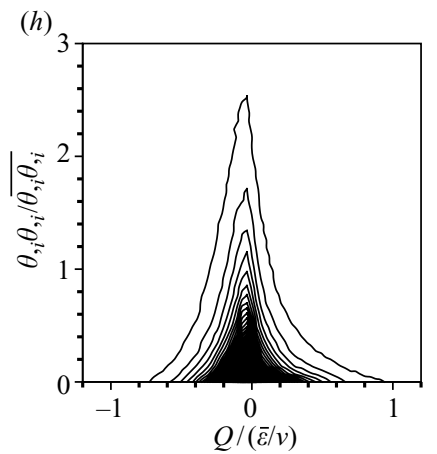

FIGURE 16. Joint p.d.f.s for $R e_{\tau}=640$ : (a), (b) $\omega_{i} \omega_{i}$ and $\varepsilon ;(c),(d) \omega_{i} \omega_{i}$ and $\theta,_{i} \theta,,_{i}$; $(e),(f) \varepsilon$ and $\theta,,_{i} \theta,,_{i} ;(g),(h) Q$ and $\theta,,_{i} \theta,{ }_{i}$. The locations examined are at $y^{+}=10((a)$, $(c),(e),(g))$ and $y / \delta=1((b),(d),(f),(h))$. The line increment is 0.1 .

here) and the instantaneous visualizations (figures 13 and 14). For $y^{+}>100$, where the departure from isotropy is nearly constant (figure $2 b$ ), the p.d.f.s are almost similar to those for isotropic turbulence (e.g. Ruetsch \& Maxey 1992; Jiménez et al. 1993; Pumir 1994; Antonia \& Orlandi 2003) and grid turbulence (Zhou \& Antonia 2000). In this region, the most intense $\theta,{ }_{i} \theta,{ }_{i}$ occurs where $\omega_{i} \omega_{i}$ is negligible but $\varepsilon$ shows small values, whilst moderate values of $\theta,{ }_{i} \theta,{ }_{i}$ tend to coincide with regions in which $\omega_{i} \omega_{i}$ is large (figure 16c-f). These considerations suggest that $\theta,{ }_{i} \theta,{ }_{i}$ is associated with $\omega_{i} \omega_{i}$ as well as $\varepsilon$ throughout the channel, although the magnitudes of the correlations vary across the flow.

In the near-wall region, $Q<0$ (strain is dominant) is not the sole contributor to $\theta,{ }_{i} \theta,{ }_{i}$. The near-wall joint p.d.f. (figure $16 \mathrm{~g}$ ) shows that $Q>0$ (vorticity is dominant) is associated with moderate magnitudes of $\theta,{ }_{i} \theta,{ }_{i}$. Also, $\rho_{\theta, i}{ }^{2} Q$ (not shown here) exhibits 
a peak at $y^{+}=10$, where $\omega_{1}$ is important for the mixing ( $\operatorname{see} \overline{\omega_{1} \theta, 3} / \omega_{1}^{\prime} \theta,_{3}^{\prime}$ in figure $7 a$ ), and its magnitude is nearly zero, indicating that the contribution of $Q>0$ to $\theta,{ }_{i} \theta,{ }_{i}$ is almost equal to that of $Q<0$. These results suggest that both quasi-streamwise vortices $(Q>0)$ and straining motions $(Q<0)$, possibly induced by the streaks as discussed in $\S 5$ (see also figure 13), are correlated with $\theta,,_{i} \theta,,_{i}$. In the outer region $\theta,{ }_{i} \theta,{ }_{i}$ is associated with $Q<0$ (the strain is dominant), as in isotropic turbulence (Brethouwer et al. 2003). This result implies that $\theta,,_{i} \theta,{ }_{i}$ is not directly related to vortical motions (e.g. transverse vortices) but to straining motions induced by the vortices and also the large-scale $\theta$ structures (see figure 14).

\section{Conclusions}

The present study has focused on conventional statistics for the vorticity and scalar derivative vectors, using DNS databases for a fully developed turbulent channel flow with a constant time-averaged heat-flux boundary condition. The data were obtained at $\operatorname{Pr}=0.71$ and 3 values of the Reynolds number $\left(R e_{\tau}=180,395\right.$ and 640$)$; for the largest Reynolds number, the spatial resolution is considered adequate for resolving the smallest scales of interest. Particular attention has been given to the correlation between the two vectors in both inner and outer regions of the flow. The salient conclusions are given below for each region:

(a) Inner region

(i) The similarity between $\overline{\omega_{2}^{+} \omega_{2}^{+}}$and $\overline{\theta,{ }_{3}^{+} \theta,{ }_{3}^{+}}$as well as between $\overline{\omega_{3}^{+} \omega_{3}^{+}}$and $\overline{\theta,,_{2}^{+} \theta,{ }_{2}^{+}}$ is excellent and reflects the strong near-wall correlation between $u_{1}$ and $\theta$. There is also close similarity between the transport equations of $\overline{\omega_{i} \omega_{i}}$ and $\overline{\theta,{ }_{i} \theta,{ }_{i}}$ in spite of a discernible difference very close to the wall arising from the difference in boundary conditions between the velocity and scalar fields.

(ii) The magnitudes of $\overline{\omega_{1}^{+} \omega_{1}^{+}}, \overline{\omega_{3}^{+} \omega_{3}^{+}}$and $\overline{\theta,{ }_{2}^{+} \theta,{ }_{2}^{+}}$increase significantly very close to the wall, as $R e_{\tau}$ increases, mainly as the result of straining induced by the wall. By contrast, the approximate independence on $R e_{\tau}$ of $\overline{\theta,{ }_{2}^{+} \theta,{ }_{2}^{+}}$and $\overline{\theta,{ }_{3}^{+} \theta,_{3}^{+}}$seems consistent with the independence on $R e_{\tau}$ of the spanwise streak spacing. Two-point correlations of $\omega_{2}$ and $\theta, 3$ along the spanwise direction have a distinct negative peak that is less dependent on $R e_{\tau}$ than the peak observed in the spanwise two-point correlations for either $u_{1}$ or $\theta$. This peak corresponds to an average width for either low-speed or low-temperature streaks of about 30 wall units.

(iii) In the transport equations of $\overline{\omega_{i} \omega_{i}}$ and $\overline{\theta,{ }_{i} \theta,,_{i}}$, the magnitude of the mean gradient production terms is of the same order as that of the turbulent production terms. The latter terms have a preference to align with the mean strain rate. (Note that unlike $2 \overline{\omega_{i} \omega_{j} s_{i j}}$, the mean strain rate is not necessarily associated with the increase in $-2 \overline{\theta,{ }_{i} \theta,{ }_{j} s_{i j}}$, since the latter is only amplified by the compressive strain rate.) The same is true for $\omega_{i}$ and $\theta,{ }_{i}$. The preferred alignment of $\omega_{i}$ is with the direction of the intermediate strain rate, whilst that of $\theta,{ }_{i}$ is at $45^{\circ}$ to the extensional and compressive strain rates respectively. Also, $\theta,{ }_{i}$ is more likely to be correlated with the compressive strain rate than in isotropic turbulence, possibly due to the space-filling and persistent features of the near-wall structures as well as the presence of the mean velocity and temperature gradients.

(iv) The magnitudes of the correlation coefficients for $\overline{\omega_{1} \theta, 3}, \overline{\omega_{2} \theta, 3}, \overline{\omega_{3} \theta, 1}, \overline{\omega_{3} \theta, 2}$ are quite large. Streaks, quasi-streamwise vortices and internal shear layers all contribute to these correlations albeit in different manners and amounts.

(v) Streaks, internal shear layers and quasi-streamwise vortices are intrinsic topological elements of the scalar dissipation rate and are important for implementing 
small-scale scalar mixing. There is a strong correlation between the scalar dissipation rate, the energy dissipation rate and the enstrophy. Nearly coincident isocontours of $\omega_{i} \omega_{i}$ and $\theta,{ }_{i} \theta,{ }_{i}$ are discerned around the circumference of streaks. The local maximum near $y^{+} \simeq 30$ for the correlation between $\overline{\omega_{i} \omega_{i}}$ and $\overline{\theta,{ }_{i} \theta,{ }_{i}}$ corresponds approximately to the average height of the streaks. The effect of vorticity (4.4), which is essentially the effect of the quasi-streamwise vortices $\left(\omega_{1}\right)$, cannot be neglected. Nor can the effect due to the strain rate, possibly induced by the streaks.

(b) Outer region

(i) Each component of $\overline{\omega_{i}^{+} \omega_{i}^{+}}$and $\overline{\theta,{ }_{i}^{+} \theta,{ }_{i}^{+}}$approaches local axisymmetry for $y^{+}>60$ and 100 , respectively, the degree of anisotropy being likely to be associated with the mean velocity and temperature gradients. The turbulent production terms, $2 \overline{\omega_{i} \omega_{j} s_{i j}}$ (4.1) and $-2 \overline{\theta,{ }_{i} \theta,{ }_{j} s_{i j}}$ (4.2), are the sole contributors to the production process, since the effect of the vorticity (4.4) can be neglected due to the small magnitude of the mean temperature gradient.

(ii) In the transport equations of $\overline{\omega_{i} \omega_{i}}$ and $\overline{\theta,{ }_{i} \theta, i}$, the ratio of the mean gradient production to the difference between the turbulent production and the dissipation rate terms does not depend on the Reynolds number. This may be useful for modelling the transport equation of $\overline{\varepsilon_{\theta}}$.

(iii) The alignment of $\omega_{i}$ is with the direction of the intermediate strain rate, while $\theta,{ }_{i}$ is along the compressive strain rate direction. The result agrees with that obtained from DNSs for box turbulence with or without an imposed mean scalar gradient.

(iv) The correlation coefficients between $\omega_{i}$ and $\theta,{ }_{i}$ and between $\omega_{i} \omega_{i}$ and $\theta,{ }_{i} \theta,{ }_{i}$, which are, by and large, caused by the presence of fronts or backs, are smaller than in the wall region. Consistently, the similarity between $u_{1}$ and $\theta$ large-scale structures is reduced compared to that between the near-wall $u_{1}$ and $\theta$ structures.

(v) Like enstrophy, the scalar dissipation rate has many characteristics in common with other turbulent flows, e.g. homogeneous isotropic turbulence, homogeneous sheared turbulence, grid turbulence and turbulent shear flows. The dominant structures are sheetlike in shape and aligned with the compressive strain rate. Their average thickness is approximately $6 \eta$ or $7 \eta_{B}$ or $y^{+}>100$ and is unlikely to be affected by the Reynolds number. The scalar dissipation rate is better correlated with the energy dissipation rate than the enstrophy.

Overall, the present results indicate that whilst the correlations in the outer region between the two small-scale fields exhibit similar features to those previously reported for homogeneous flows, the correlations near the wall are quite different. In particular, the large magnitudes of these correlations underline the important potential role of the organized structures for enhancing small-scale mixing.

Computations performed on Numerical Simulator III at the Computer Centre of the Japan Aerospace Exploration Agency are gratefully acknowledged. The authors are grateful to the referees for their precise comments. HA was partially supported by the Ministry of Education, Science, Sports and Culture's Grant-in-Aid for Young Scientists (B), 20760125, 2008. RAA acknowledges the support of the Australian Research Council.

\section{Appendix. Validation of the computational accuracy}

A few checks of the computational accuracy are provided by distributions at $y / \delta=1$ of $k_{x}^{4} \phi_{u}\left(k_{x}\right)$ and $k_{z}^{4} \phi_{u}\left(k_{z}\right)$ (figure $\left.17 a, c\right)$, normalized by the Kolmogorov scales $\left(v_{K}, \eta\right.$ ), and $k_{x}^{4} \phi_{\theta}\left(k_{x}\right)$ and $k_{z}^{4} \phi_{\theta}\left(k_{z}\right)$ (figure $\left.17 b, d\right)$, normalized by the Batchelor scales $\left(\theta_{B}, \eta_{B}\right)$ 

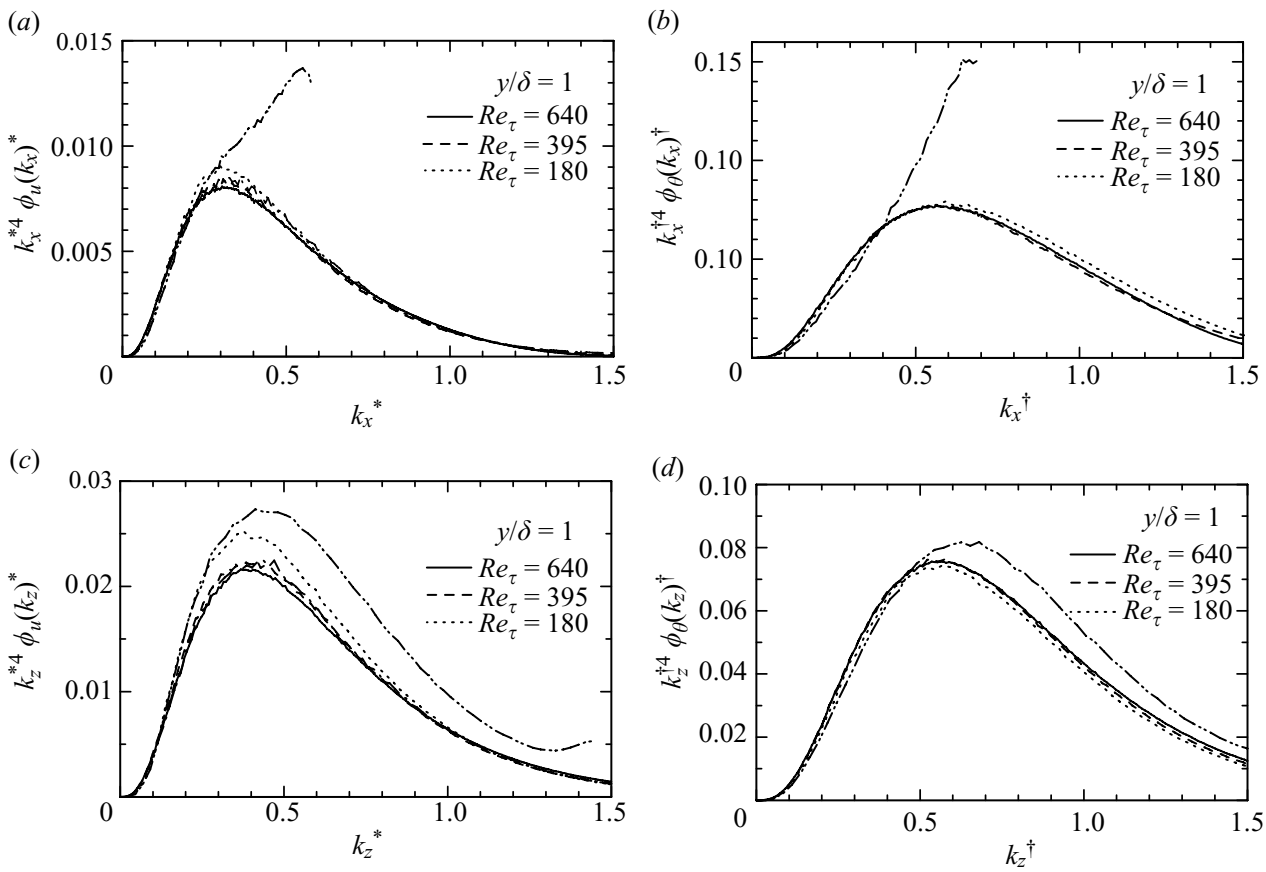

FiguRE 17. Streamwise and spanwise spectra of $u_{1}$ and $\theta$ multiplied by $k_{x}^{4}$ or $k_{z}^{4}$ at $y / \delta=1$ normalized by the Kolmogorov scales $\left(v_{K}\right.$ and $\left.\eta\right)$ and the Batchelor scales $\left(\theta_{B}\right.$ and $\left.\eta_{B}\right):(a) \quad k_{x}^{* 4} \phi_{u}\left(k_{x}\right)^{*} ;(b) \quad k_{x}^{\dagger 4} \phi_{\theta}\left(k_{x}\right)^{\dagger} ;(c) \quad k_{z}^{* 4} \phi_{u}\left(k_{z}\right)^{*} ;(d) \quad k_{z}^{\dagger 4} \phi_{\theta}\left(k_{z}\right)^{\dagger} ;-\cdot-\cdot-$. Moser et al. (1999) for $\operatorname{Re}_{\tau}=590$ at $y / \delta=1 ;-\cdots-\cdots-$, Kasagi et al. (1992) for $\operatorname{Re}_{\tau}=150$ at $y / \delta=1$.

denoted by the superscript $\dagger$. The streamwise and spanwise spectra of $u_{1}$ and $\theta$ are defined such that

$$
\begin{aligned}
\overline{u_{1} u_{1}} & =\int_{0}^{\infty} \phi_{u}\left(k_{x}\right) \mathrm{d} k_{x}=\int_{0}^{\infty} \phi_{u}\left(k_{z}\right) \mathrm{d} k_{z}, \\
\overline{\theta \theta} & =\int_{0}^{\infty} \phi_{\theta}\left(k_{x}\right) \mathrm{d} k_{x}=\int_{0}^{\infty} \phi_{\theta}\left(k_{z}\right) \mathrm{d} k_{z},
\end{aligned}
$$

where $\phi_{u}$ and $\phi_{\theta}$ are the spectra of $u_{1}$ and $\theta$, respectively, and $k_{x}$ and $k_{z}$ the streamwise and spanwise wavenumbers, respectively. For comparison, the spectra of Moser et al. (1999) and Kasagi et al. (1992) at the centreline, which were obtained with spectral methods, are included in figure 17. There is no energy pile-up in the present streamwise and spanwise spectra multiplied by $k_{x}^{4}$ and $k_{z}^{4}$ at high wavenumbers. The agreement between the present spectra and those of Moser et al. (1999) is satisfactory. The poor agreement with Kasagi et al. (1992) at high wavenumbers is likely to have been caused by the insufficient spatial resolution in their simulations. The adequate closure (or convergence) of the present spectral distributions indicates that the present spatial resolution is sufficient to enable the derivatives which appear in the vorticity and scalar dissipation rate to be estimated reliably.

The transport equations of the turbulent kinetic energy, $k\left(\equiv\left(\overline{u_{1}^{2}}+\overline{u_{2}^{2}}+\overline{u_{3}^{2}}\right) / 2\right)$, and the temperature variance, $k_{\theta}\left(\equiv \overline{\theta^{2}} / 2\right)$, provide a useful indirect check of how well the 
(a)

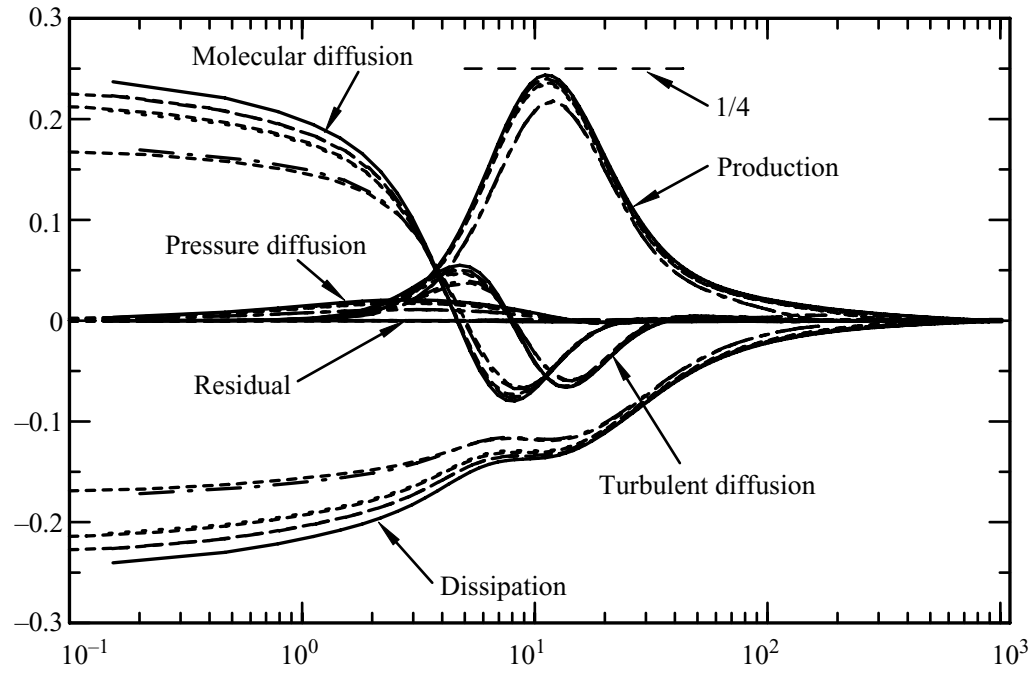

(b)

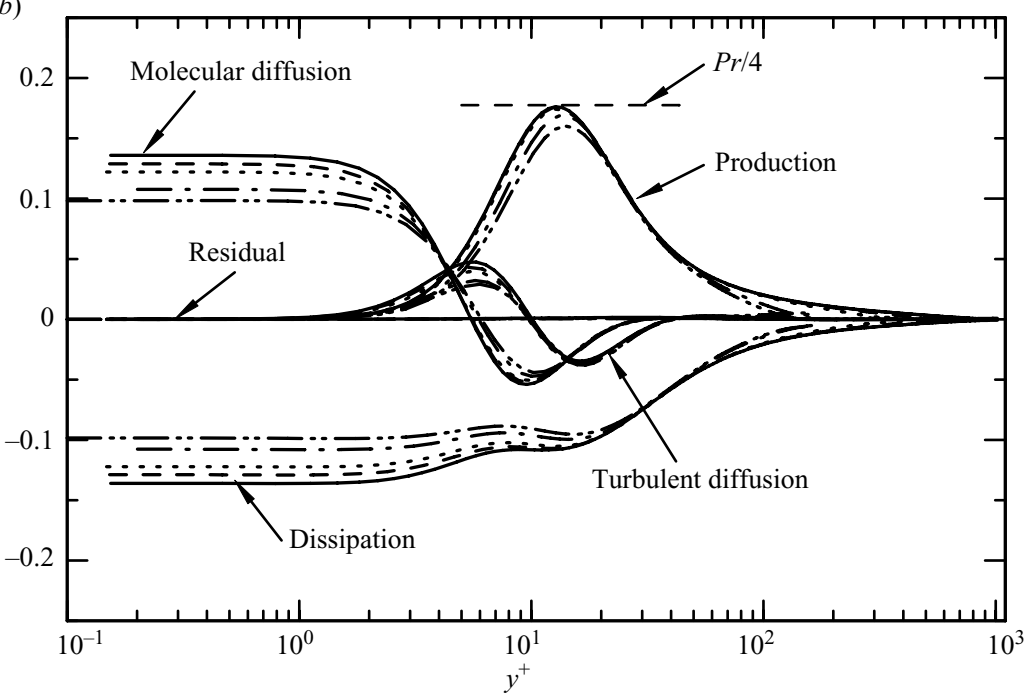

FIGURE 18. Budgets of the turbulent kinetic energy and the temperature variance for $R e_{\tau}=$ 180, 395 and 640: (a) $k$; (b) $k_{\theta},---, R e_{\tau}=640 ; \cdots \cdots \cdots, R e_{\tau}=395 ;-\cdot-\cdot \cdot-\cdot R e_{\tau}=180$; - - - - Moser et al. (1999) at $R e_{\tau}=180,395$ and 590; - Abe et al. $(2004 b)$ at $R e_{\tau}=1020$; $-\cdots-\cdots-$, Kasagi et al. (1992) at $R e_{\tau}=150$.

destruction terms are estimated. The transport equation of $k$, normalized by $u_{\tau}^{4} / v$ is

$$
0=\underbrace{-\overline{u_{i}^{+} u_{k}^{+}} \frac{\partial \bar{U}_{i}^{+}}{\partial x_{k}^{+}}}_{1} \underbrace{-\frac{\partial}{\partial x_{k}^{+}}\left(\frac{1}{2} \overline{u_{i}^{+2} u_{k}^{+}}\right)}_{2} \underbrace{-\frac{\partial}{\partial x_{i}^{+}}\left(\frac{1}{2} \overline{u_{i}^{+} p^{+}}\right)}_{3}+\underbrace{+\frac{\partial^{2}}{\partial x_{k}^{+2}}\left(\frac{1 \overline{u_{i}^{+2}}}{2}\right)}_{4}-\underbrace{\overline{\left(\frac{\partial u_{i}^{+}}{\partial x_{k}^{+}}\right)^{2}}}_{5},
$$

where terms 1, 2, 3, 4, 5 denote the production, turbulent diffusion, pressure diffusion, molecular diffusion and homogeneous dissipation rate, respectively. Also, 
the transport equation of $k_{\theta}$, normalized by $u_{\tau}^{2} T_{\tau}^{2} / v$, is

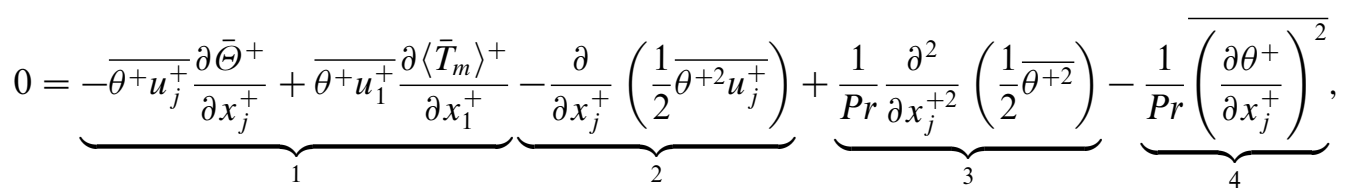

where terms 1, 2, 3, 4 are the production, turbulent diffusion, molecular diffusion and dissipation, respectively. Note that in (A 3), the homogeneous dissipation $\bar{\varepsilon}_{\text {hom }}$ is used instead of the full epsilon $\bar{\varepsilon}$. Figure $18(a, b)$ shows the budgets of $k$ and $k_{\theta}$ respectively; the data of Abe et al. (2004b) at $R e_{\tau}=1020$ and $\operatorname{Pr}=0.71$ have been included. Agreement with the existing DNS data (the data of Kasagi et al. 1992 at $R e_{\tau}=150$ and $P r=0.71$ and Moser et al. 1999 at $R e_{\tau}=180,395$ and 590) is quite good. The significant $R e$ dependence in the near-wall region conforms with the low $R e$ effects reported by Antonia \& Kim (1994a). The maximum value of the production reaches 0.243 at $R e_{\tau}=1020$, which is very close to its asymptotic value $(1 / 4)$. The distributions in figure $18(b)$ are very similar to those in figure $18(a)$, notwithstanding the absence of the pressure in (A 4). For $R e_{\tau} \geqslant 395$, the magnitude of the production is $\operatorname{Pr} / 4$, which is the theoretical maximum value. This suggests that the thermal field reaches local equilibrium at a smaller $R e_{\tau}$ than the velocity field.

\section{REFERENCES}

Abe, H., Kawamura, H. \& Matsuo, Y. 2001 Direct numerical simulation of a fully developed turbulent channel flow with respect to the Reynolds number dependence. ASME J. Fluids Engng 123, 382-393.

Abe, H., Kawamura, H. \& Choi, H. 2004a Very large-scale structures and their effects on the wall shear-stress fluctuations in a turbulent channel flow up to $R e_{\tau}=640$. ASME J. Fluids Engng 126, 835-843.

Abe, H., Kawamura, H. \& Matsuo, Y. 2004b Surface heat-flux fluctuations in a turbulent channel flow up to $R e_{\tau}=1020$ with $P r=0.025$ and 0.71. Intl J. Heat Fluid Flow 25, 404-419.

Antonia, R. A., Abe, H. \& Kawamura, H. 2008 Analogy between velocity and scalar fields in a turbulent channel flow. J. Fluid Mech. Submitted.

Antonia, R. A. \& Browne, L. W. B. 1983 The destruction of temperature fluctuations in a turbulent plane jet. J. Fluid Mech. 134, 67-83.

Antonia, R. A. \& Chambers, A. J. 1980 On the correlation between turbulent velocity and temperature derivatives in the atmospheric surface layer. Boundary-Layer Meteorol. 18, 399410.

Antonia, R. A., Chambers, A. J., Britz, D. \& Browne, L. W. B. 1986 Organized structures in a turbulent plane jet: topology and contribution to momentum and heat transport. J. Fluid Mech. 172, 211-229.

Antonia, R. A. \& Kim, J. 1991 Turbulent Prandtl number in the near-wall region of a turbulent channel flow. Intl J. Heat Mass Transfer 34 (7), 1905-1908.

Antonia, R. A. \& Kim, J. $1994 a$ Low-Reynolds-number effects on near-wall turbulence. J. Fluid Mech. 276, 61-80.

Antonia, R. A. \& Kim, J. 1994b A numerical study of local isotropy of turbulence. Phys. Fluids 6 (2), 834-841.

Antonia, R. A., KIm, J. \& BRowne, L. W. B. 1991 Some characteristics of small-scale turbulence in a turbulent duct flow. J. Fluid Mech. 233, 369-388.

Antonia, R. A., Krishnamoorthy, L. V. \& Fulachier, L. 1988 Correlation between the longitudinal velocity fluctuation and temperature fluctuation in the near-wall region of a turbulent boundary layer. Intl J. Heat Mass Transfer 31 (4), 723-730. 
Antonia, R. A. \& Mi, J. 1993 Temperature dissipation in a turbulent round jet. J. Fluid Mech. 250, 531-551.

Antonia, R. A. \& Orlandi, P. 2003 Effect of Schmidt number on small-scale passive scalar turbulence. Appl. Mech. Rev. 56 (6), 615-632.

Antonia, R. A. \& Orlandi, P. 2004 Similarity of decaying isotropic turbulence with a passive scalar. J. Fluid Mech. 505, 123-151.

Antonia, R. A. \& VAN AtTA, C. W. 1975 On the correlation between temperature and velocity dissipation fields in a heated turbulent jet. J. Fluid Mech. 67, 273-288.

Antonia, R. A. \& Van AttA, C. W. 1979 Skewness of spatial derivatives of temperature in a turbulent boundary layer. Phys. Fluids 22 (12), 2430-2431.

Ashurst, W. T., Kerstein, A. R., Kerr, R. M. \& Gibson, C. H. 1987 Alignment of vorticity and scalar gradient with strain rate in simulated Navier-Stokes turbulence. Phys. Fluids 30, 2343-2353.

Batchelor, G. K. 1946 The theory of axisymmetric turbulence. Proc. R. Soc. Lond. A 186, 480-502.

Blackburn, H. M., Mansour, N. N. \& Cantwell, B. J. 1996 Topology of fine-scale motions in turbulent channel flow. J. Fluid Mech. 310, 269-292.

Brethouwer, G., Hunt, J. C. R. \& Nieuwstadt, F. T. M. 2003 Micro-structure and Lagrangian statistics of the scalar field with a mean gradient in isotropic turbulence. J. Fluid Mech. 474, $193-225$.

Brown, G. L. \& Thomas, A. S. W. 1977 Large structure in a turbulent boundary layer. Phys. Fluids 20 (10), S243-S252.

Browne, L. W. B., Antonia, R. A. \& Shah, D. A. 1987 Turbulent energy dissipation in a wake. J. Fluid Mech. 179, 307-326.

Buch, K. A. \& DAHM, W. J. A. 1998 Experimental study of the fine-scale structure of conserved scalar mixing in turbulent shear flows. Part 2. $S c \approx 1$. J. Fluid Mech. 364, 1-29.

Chandrasekhar, S. 1950 The theory of axisymmetric turbulence. Proc. R. Soc. Lond. A 242, 557-577.

Chen, C. P. \& Blackwelder, R. F. 1978 Large-scale motion in a turbulent boundary layer: a study using temperature contamination. J. Fluid Mech. 89, 1-31.

Corrsin, S. 1953 Remarks on turbulent heat transfer: an account of some features of the phenomenon in fully turbulent regions. In Proc. of the first Iowa Thermodynamics Symposium, pp. 5-30, State University of Iowa, Iowa City.

George, W. K. \& Hussein, H. J. 1991 Locally axisymmetric turbulence. J. Fluid Mech. 233, 1-23.

GonZaLEZ, M. 2002 Effect of vorticity on second- and third-order statistics of passive scalar gradients. Phys. Rev. E 60, 056307.

Holzer, M. \& Siggia, E. D. 1994 Turbulent mixing of a passive scalar. Phys. Fluids 6, 1820-1837.

Horiuti, K. 2001 A classification method for vortex sheet and tube structures in turbulent flows. Phys. Fluids 13, 3756-3774.

Iritani, Y., Kasagi, N. \& Hirata, M. 1985 Heat transfer mechanism and associated turbulence structure in the near-wall region of a turbulent boundary layer. In Turbulent Shear Flows 4 (ed. L. J. S. Bradbury, F. Durst, B. E. Launder, F. W. Schmidt and J. H. Whitelaw), pp. 223-234, Springer.

Iwamoto, K., KaSAGI, N. \& SuzUKi, Y. 2005 Direct numerical simulation of turbulent channel flow at $R e_{\tau}=2320$. In Proc. of the 6th Symp. on Smart Control of Turbulence, pp. 327-333, Tokyo.

JimÉneZ, J. 1992 Kinematic alignment effects in turbulent flows. Phys. Fluids A 4, 652-654.

JimÉnez, J., Del Álamo, J. C. \& Flores, O. 2004 The large scale dynamics near-wall turbulence. J. Fluid Mech. 505, 179-199.

JimÉnez, J., Moin, P., Moser, R. \& KeEFe, L. 1988 Ejection mechanism in the sublayer of a turbulent channel flow. Phys. Fluids 31, 1311-1313.

Jiménez, J., Wray, A. A., Saffmann, P. G. \& Rogallo R. S. 1993 The structure of intense vorticity in isotropic turbulence. J. Fluid Mech. 255, 65-90.

Johansson, A. V., Alfredsson, P. H. \& Kim, J. 1991 Evolution and dynamics of shear-layer structure in near-wall turbulence. J. Fluid. Mech. 224, 579-599.

Johansson, A. V. \& WikströM, P. M. 1999 DNS and modelling of passive scalar transport in turbulent channel flow with a focus on scalar dissipation rate modelling. Flow Turb. Combust. 63, 223-245.

Kasagi, N. Tomita, Y. \& Kuroda, A. 1992 Direct numerical simulation of passive scalar field in a turbulent channel flow. ASME J. Heat Transfer 144, 598-606. 
Kasagi, N. \& Ohtsubo, Y. 1993 Direct numerical simulation of low Prandtl number thermal field in a turbulent channel flow. In Turbulent Shear Flows 8 (ed. F. Durst, R. Friedrich, B. E. Launder, F. W. Schmidt, U. Schumann and J. H. Whitelaw), pp. 97-119, Springer.

Kawamura, H., Abe, H. \& Matsuo, Y. 1999 DNS of turbulent heat transfer in channel flow with respect to Reynolds and Prandtl number effects. Intl J. Heat Fluid Flow 20, 196-207.

Kawamura, H., Abe, H., Matsuo, Y. \& Choi, H. 2002 Large-scale structures of velocity and scalar fields in turbulent channel flows. In Intl Symp. on Dynamics and Statistics of Coherent Structures in Turbulence: Roles of Elementary Vortices, pp. 49-64, Tokyo.

Kawamura, H. Abe, H. \& Shingai, K. 2000 DNS of turbulence and heat transport in a channel flow with different Reynolds and Prandtl numbers and boundary conditions. In Proc. of the 3rd Intl Symp. on Turbulence, Heat and Mass Transfer (ed. Y. Nagano, K. Hanjalic and T. Tsuji), pp. 15-32, Aichi Shuppan.

Kawamura, H., Ohsaka, K., Авe, H. \& Yамamoto, K. 1998 DNS of turbulent heat transfer in channel flow with low to medium-high Prandtl number fluid. Intl J. Heat Fluid Flow 19, 482-491.

KERR, R. M. 1985 Higher-order derivative correlations and the alignment of small-scale structures in isotropic numerical turbulence. J. Fluid Mech. 153, 31-58.

KIM, J. 1988 Investigation of heat and mass transport in turbulent flows via numerical simulation. In Transport Phenomena in Turbulent Flows: Theory, Experiment and Numerical Simulation (ed. M. Hirata \& N. Kasagi), pp. 157-170. Hemisphere Publishing.

KIM, J. \& MoIN. P. 1989 Transport of passive scalars in a turbulent channel flow. In Turbulent shear flows 6 (ed. J.-C. André, J. Cousteix, F. Durst, B. E. Launder, F. W. Schmidt and J. H. Whitelaw), pp. 85-96, Springer.

Kim, J., Morn. P. \& Moser, R. 1987 Turbulence statistics in fully developed channel flow at low Reynolds number. J. Fluid Mech. 177, 133-166.

Kravchenko, A. G. \& Moin, P. 1997 On the effect of numerical errors in large eddy simulations of turbulent flows. J. Comput. Phys. 131, 310-322.

KrishnAmOORTHY, L. V. \& ANTONiA, R. A. 1987 Temperature-dissipation measurements in a turbulent boundary layer. J. Fluid Mech. 176, 265-281.

MoIN, P. \& KIM, J. 1985 The structure of the vorticity field in turbulent channel flow. Part 1. Analysis of instantaneous fields and statistical correlatins. J. Fluid. Mech. 155, 441-464.

Moin, P. \& Mahesh, K. 1998 Direct numerical simulation: a tool in turbulence research. Annu. Rev. Fluid Mech. 30, 539-578.

Moin, P., Adrian, R. \& Kim, J. 1987 Stochastic estimation of organized structures in turbulent channel flow. In Proc. of the 6th Symp. on Turbulent Shear Flows, pp. 16-9-1-16-9-8, Toulouse, France.

Moisy, F., Willaime, H., Andersen, J. S. \& Tabeling, P. 2001 Passive scalar intermittency in low temperature helium flows. Phys. Rev. Lett. 86, 4827-4830.

Morinishi, Y., Lund, T. S., VAsilyev, O. V. \& Moin, P. 1998 Fully conservative higher order finite difference schemes for incompressible flow. J. Comput. Phys. 143, 90-124.

Moser, R. D., Kim, J. \& Mansour, N. N. 1999 Direct numerical simulation of turbulent channel flow up to $R e_{\tau}=590$. Phys. Fluids 11, 943-945.

Nagano, Y. \& KIM, C. 1988 A two-equation model for heat transport in wall turbulent shear flows. ASME J. Heat Transfer 110, 583-589.

Namazian, M., Schefer, R. W. \& Kelly, J. 1988 Scalar dissipation measurements in the developing region of a jet. Comb. Flame 74, 147-160.

Nomura, K. K. \& Elghobashi, S. E. 1992 Mixing characteristics of an inhomogeneous scalar in isotropic and homogeneous sheared turbulence. Phys. Fluids A 4 (3), 606-625.

Orlandi, P., LeONARdi, S. \& ANTONia, R. A. 2006 Vortex structures in a rough-wall channel flow and their influence on passive scalar. In Proc. of IUTAM Symposium on Elementary Vortices and Coherent Structures: Significance in Turbulence Dynamics (ed. S. Kida), pp. 39-49, Springer.

ÖstERLund, J. M. 1999 Experimental studies of zero pressure-gradient turbulent boundary layer Flow. PhD thesis, Royal Institute of Technology, Stockholm.

Peters, N. 2000 Turbulent Combustion. Cambridge University Press.

Prasad, R. R. \& SReEnivasan, K. R. 1990 Quantitative three-dimensional imaging and the structure of passive scalar fields in fully turbulent flows. J. Fluid Mech. 216, 1-34.

Pumir, A. 1994 A numerical study of the mixing of a passive scalar in three dimensions in the presence of a mean gradient. Phys. Fluids 6 (6), 2118-2132. 
Robinson, S. K. 1991 The kinematics of turbulent boundary layer structure. Tech Rep. TM103859. NASA.

Rodi, W. \& Mansour, N. N. 1993 Low Reynolds number $k-\varepsilon$ modelling with the aid of direct simulation data. J. Fluid Mech. 250, 509-529.

RuetsCh, G. R. \& MAXEY, M. R. 1991 Small-scale features of vorticity and passive scalar fields in homogeneous isotropic turbulence. Phys. Fluids A 3, 1587-1597.

Ruetsch, G. R. \& MAXEY, M. R. 1992 The evolution of small-scale structures in homogeneous isotropic turbulence. Phys. Fluids A 4, 2747-2760.

She, Z.-S., JACKSON E. \& OrsZAG, S. A. 1990 Intermittent vortex structures in homogeneous isotropic turbulence. Nature 344, 226-228.

Smith, C. R. \& Metzler, S. P. 1983 The characteristics of low-speed streaks in the near-wall region of a turbulent boundary layer. J. Fluid Mech. 129, 27-54.

SpalaRT, P. R. 1988 Direct simulation of a turbulent boundary layer up to $R_{\theta}=1410$. J. Fluid Mech. 187, 61-98.

Spalart, P. R., Moser, R. D. \& Rogers, M. M. 1991 Spectral methods for the Navier-Stokes equations with one infinite and two periodic directions. J. Comp. Phys. 96, 297-324.

Sreenivasan, K. R., Antonia, R. A. \& Britz, D. 1979 Local isotropy and large structures in a heated turbulent jet. J. Fluid Mech. 94, 745-775.

Sreenivasan, K. R., Antonia, R. A. \& Danh, H. Q. 1977 Temperature dissipation fluctuations in a turbulent boundary layer. Phys. Fluids 20 (8), 1238-1249.

Sreenivasan, K. R. 1990 Turbulence and the tube. Nature 344, 192-193.

Sreenivasan, K. R. 1991 On local isotropy of passive scalars in turbulent shear flows. Proc. R. Soc. Lond. A 434, 165-182.

Sreenivasan, K. R. \& Antonia, R. A. 1997 The phenomenology of small-scale turbulence. Annu. Rev. Fluid Mech. 29, 435-472.

Su, L. K. \& Clemens, N. T. 2003 The structure of fine-scale scalar mixing in gas-phase planar turbulent jets. J. Fluid Mech. 488, 1-29.

Tanahashi, M., Kang, S.-J., Miyamoto, T., Shiokawa, S. \& Miyauchi, T. 2004 Scaling law of fine scale eddies in turbulent channel flows up to $R e_{\tau}=800$. Intl J. Heat Fluid Flow 25, 331-340.

TAVOUlaris, S. \& CoRrsin, S. 1981 Experiments in nearly homogeneous shear flow with a uniform mean temperature gradient. Part 2. The fine structure. J. Fluid Mech. 104, 349-367.

Tennekes, H. \& Lumley, J. L. 1972 A First Course in Turbulence. The MIT press.

Vedula, P., Yeung, P. K. \& Fox, R. O. 2001 Dynamics of scalar dissipation in isotropic turbulence: a numerical and modelling study. J. Fluid Mech. 433, 29-60.

Vincent, A. \& Meneguzzi, M. 1994 The dynamics of vorticity tubes in homogeneous turbulence. J. Fluid Mech. 258, 245-254.

Wang, L.-P., Chen, S. \& Brasseur, J. G. 1999 Examination of hypothesis in the Kolmogorov refined turbulence theory through high-resolution simulations. Part 2. Passive scalar field. $J$. Fluid Mech. 400, 163-197.

Warhaft, Z. 2000 Passive scalars in turbulent flows. Annu. Rev. Fluid Mech. 32, 203-240.

Watanabe, T. \& Gotoh, T. 2004 Statistics of a passive scalar in homogeneous turbulence. New J. Phys. 6, 40. http://www.njp.org/.

Watanabe, T. \& GotoH, T. 2007 Inertial-range intermittency and accuracy of direct numerical simulation for turbulence and passive scalar turbulence. J. Fluid Mech. 590, 117-146.

YEUNG, P. K. 2001 Lagrangian characteristics of turbulence and scalar transport in direct numerical simulations. J. Fluid Mech. 427, 241-274.

Yeung, P. K., Donzis, D. A. \& Sreenivasan, K. R. 2005 High-Reynolds-number simulation of turbulent mixing. Phys. Fluids 17, 081703.

Yeung, P. K., Xu, S. \& Sreenivasan, K. R. 2002 Schmidt number effects on turbulent transport with uniform mean scalar gradient. Phys. Fluids 14 (12), 4178-4191.

Yoshizawa, A. 1988 Statistical modelling of passive-scalar diffusion in turbulent shear flows. $J$. Fluid Mech. 195, 541-555.

Zhou, T. \& Antonia, R. A. 2000 Approximations for turbulent kinetic energy and temperature variance dissipation rates in grid turbulence. Phys. Fluids 12, 335-344. 